\title{
Moment maps and equivariant volumes
}

\author{
Alberto Della Vedova and Roberto Paoletti *
}

\section{Introduction}

Let $M$ an n-dimensional complex projective variety, and consider a holomorphic line bundle $L$ on $M$. The volume of $L$ is defined as

$$
\operatorname{vol}(L)=: \limsup _{k \rightarrow+\infty} \frac{\mathrm{n} !}{k^{\mathrm{n}}} \operatorname{dim} H^{0}\left(M, L^{\otimes k}\right) .
$$

In the special case where $L$ is ample (or, more generally, nef and big) $\operatorname{vol}(L)$ is simply the top self-intersection of $L$, but in general the study of the volume of arbitrary big line bundles has proven to be a subtle and rich subject (see [F], [DEL], and [L] for a complete discussion, examples, and references).

Suppose now that $G$ is a compact connected g-dimensional Lie group acting holomorphically on $M$ (one might equivalently start from a reductive complex Lie group $\tilde{G}$, but given the prominent role of moment maps in our discussion we shall mostly work with a fixed maximal compact subgroup $G \subseteq \tilde{G})$. Let us fix a maximal torus $T \subseteq G$ and a Weyl chamber in its dual Lie algebra, and let $\Lambda_{+}=\{\mu\}$ be the corresponding set of highest weights for $G$. For every highest weight $\mu$, let $V_{\mu}$ be the finite-dimensional irreducible representation of $G$ associated to $\mu$. If the action of $G$ linearizes to a line bundle $L$ on $M$, for every integer $k \geq 0$ there is an induced linear action of $G$ on the space of global holomorphic sections $H^{0}\left(M, L^{\otimes k}\right)$. For every $k \geq 0$, therefore, we have a $G$-equivariant direct sum decomposition

$$
H^{0}\left(M, L^{\otimes k}\right)=\bigoplus_{\mu} H^{0}\left(M, L^{\otimes k}\right)_{\mu}
$$

where for every $\mu$ the summand $H^{0}\left(M, L^{\otimes k}\right)_{\mu}$ is $G$-equivariantly isomorphic to a direct sum of copies of $V_{\mu}: H^{0}\left(M, L^{\otimes k}\right)_{\mu} \cong V_{\mu}^{\oplus N^{(k)}(\mu)}$ as $G$-modules. In

*Address. Dipartimento di Matematica e Applicazioni, Università degli Studi di Milano Bicocca, Via R. Cozzi 53, 20125 Milano, Italy; e-mail: alberto.dellavedova@unimib.it, roberto.paoletti@unimib.it 
the ample case, the relation between the algebraic structure of the decomposition (2), expressed by the multiplicities $N^{(k)}(\mu)$, and the Hamiltonian geometry of the linearization, expressed by an associated moment map, is the object of the circle of ideas revolving around the general principle quantization commutes with reduction; we refer to [GS1], JJ], [Ki3], [M2], [MS], $[\mathrm{S}$, GGK]. In the algebraic context, the asymptotic properties of equivariant modules have been studied in [BD, B]. For finite group actions, similar questions have been considered in [P1].

Now, given that the expected dimension of the quotient of $M$ by $G$ is $\mathrm{n}-\mathrm{g}$, we are led to introduce and study equivariant volumes

$$
\operatorname{vol}_{\mu}(L)=: \limsup _{k \rightarrow+\infty} \frac{(\mathrm{n}-\mathrm{g}) !}{k^{\mathrm{n}-\mathrm{g}}} \operatorname{dim} H^{0}\left(M, L^{\otimes k}\right)_{\mu} .
$$

A number of questions are naturally posed: For example, is $\operatorname{vol}_{\mu}(L)$ always finite? Is it homogeneous? Can it be geometrically determined in terms of appropriate top self-intersections, as in the ample action-free case? Does it only depend on the numerical equivalence class of the $G$-linearized line bundle $L$ ? If so, does it determine a continuous functions on the $G$-NéronSeveri space of $M, \mathrm{NS}^{G}(M)_{\mathbb{R}}$, and does an estimate similar to the one in Theorem 2.2.44 of [L] still hold? Also, is there an equivariant version of Fujita approximation for big classes?

The equivariant setting makes these problems not trivial even when $L$ is ample. In general, for instance, $\operatorname{vol}_{\mu}(L)$ may well be infinite for many $\mu$ 's or identically zero for every $\mu$.

In this article, after determining equivariant volumes for ample and regular (meaning that the stable and semi-stable loci coincide, and are nonempty) $G$-linearized line bundles on $M$ by use of the Riemann-Roch formulae for multiplicities (GS1], [M2], [JK]), we shall use ideas and techniques from the algebro-geometric theory of action-free volumes (especially from $\S 2$ of [L]) and well-known facts from GIT and the theory of moment maps (GS1], [DH], [Ki1, Ki2, [Th]) to give some answers to the previous questions.

\section{Preliminaries.}

Throughout this article, $G$ will denote a g-dimensional compact connected Lie group, and $\widetilde{G}$ will be its complexification, a complex reductive Lie group in which $G$ sits a maximal compact subgroup.

As is well-known, an holomorphic action of $G$ on a complex projective variety $M$ extends to a holomorphic action of $\widetilde{G}$ on $M$, and if the $G$-action

linearizes to a line bundle $L$, then so does the action of $\widetilde{G}$ GS1. We shall 
briefly say that $M$ is a (complex) projective $G$-variety (or $\widetilde{G}$-variety), and $L$ a $G$-linearized line bundle on $M$. After $[\mathrm{DH}]$, we shall denote by $\operatorname{Pic}^{G}(M)$ the group of all isomorphisms classes of $G$-linearized line bundles on $M$.

If $L \in \operatorname{Pic}^{G}(M)$ is ample and has non-empty semi-stable locus, we shall generally denote by $M_{0}(L)$ the GIT quotient $M / /{ }_{L} \widetilde{G}=M^{\mathrm{ss}}(L) / \widetilde{G}$.

\subsection{Homological and numerical equivalence}

Let $c_{1}^{(\mathbb{Z})}: \operatorname{Pic}(M) \rightarrow H^{2}(M, \mathbb{Z})$ be the map $c_{1}^{(\mathbb{Z})}\left(\mathcal{O}_{M}(D)\right)=:[D]_{\text {hom }}$, and let $\operatorname{Pic}(M)_{0}=: \operatorname{ker}\left(c_{1}^{(\mathbb{Z})}\right)$. Thus, $\operatorname{Pic}(M)_{0}$ parametrizes the line bundles on $M$ that are deformations of the trivial line bundle.

Next let $c_{1}^{(\mathbb{R})}: \operatorname{Pic}(M) \rightarrow H^{2}(M, \mathbb{R})$ be the composition of $c_{1}^{(\mathbb{Z})}$ with the natural map

$$
H^{2}(M, \mathbb{Z}) \rightarrow H^{2}(M, \mathbb{Z})_{\text {t.f. }}=: H^{2}(M, \mathbb{Z}) / H^{2}(M, \mathbb{Z})_{\text {tor }} \hookrightarrow H^{2}(M, \mathbb{R}) ;
$$

here $H^{2}(M, \mathbb{Z})_{\text {tor }}$ denotes the torsion part of $H^{2}(M, \mathbb{Z})$. Let $\operatorname{Pic}(M)_{0}^{\prime}=$ : $\operatorname{ker}\left(c_{1}^{(\mathbb{R})}\right)$. Thus, $L \in \operatorname{Pic}(M)_{0}^{\prime}$ if and only if $c_{1}^{(\mathbb{Z})}\left(\mathcal{O}_{M}(D)\right) \in H^{2}(M, \mathbb{Z})_{\text {tor }}$, the torsion part of $H^{2}(M, \mathbb{Z})$. Equivalently, $L \in \operatorname{Pic}(M)_{0}^{\prime}$ if and only if some tensor power $L^{\otimes m}$ is a deformation of the trivial line bundle. Since $H^{2}(M, \mathbb{Z})_{\text {tor }}$ is finite, $\operatorname{Pic}(M)_{0}^{\prime}$ consists of finitely many connected components, one for each element of $H^{2}(M, \mathbb{Z})_{\text {tor }}$, and every component is isomorphic to $\operatorname{Pic}(M)_{0}$.

Given the choice of some Hermitian structure on $L \in \operatorname{Pic}(M)$, and in view of the de Rahm theorem, we also have $c_{1}^{(\mathbb{R})}(L)=\frac{i}{2 \pi}[\Theta]$, where $[\Theta]$ is the cohomology class of the curvature of the compatible connection on $L$. Thus, $L \in \operatorname{Pic}(M)_{0}^{\prime}$ if and only if $\Theta$ is an exact form. As shown in 2.3.1 of [DH], the latter condition implies that there exists an open trivializing cover for $L$ for which the transition functions are constants of absolute value 1 .

By the proof of 2.3.3 of [DH], there is a natural choice of a $G$-linearization $L^{G}$ on any $L \in \operatorname{Pic}(M)$ that can be described by a system of constant transition functions.

Now let $f_{G}: \operatorname{Pic}^{G}(M) \rightarrow \operatorname{Pic}(M)$ be the forgetful map, which to any $G$-linearized line bundle associates the underlying line bundle (apart from this section, we shall be systematically sloppy, and use the same symbol for $L \in \operatorname{Pic}^{G}(M)$ and its image in $\left.\operatorname{Pic}(M)\right)$. By the above considerations, we have a commutative diagram of split short exact sequences:

$$
\begin{aligned}
& 1 \rightarrow \chi(G) \longrightarrow \operatorname{ker}\left(c_{1}^{(\mathbb{R})} \circ f_{G}\right) \stackrel{f_{G}}{\longrightarrow} \operatorname{Pic}(M)_{0}^{\prime} \rightarrow 0 \\
& \| \quad \uparrow \quad \uparrow \\
& 1 \rightarrow \chi(G) \longrightarrow \operatorname{ker}\left(c_{1}^{(\mathbb{Z})} \circ f_{G}\right) \stackrel{f_{G}}{\longrightarrow} \operatorname{Pic}(M)_{0} \rightarrow 0
\end{aligned}
$$


where $\chi(G)$ is the character group of $G$, the vertical arrows are inclusions, and the splitting is given by the maps $L \mapsto L^{G}$.

Definition 2.1. ([DH], Definition 2.3.4) Let us set

$$
\begin{array}{ll}
\operatorname{Pic}^{G}(M)_{0}=: & \left\{L^{G}: L \in \operatorname{Pic}(M)_{0}\right\}, \\
\operatorname{Pic}^{G}(M)_{0}^{\prime}=: & \left\{L^{G}: L \in \operatorname{Pic}(M)_{0}^{\prime}\right\} .
\end{array}
$$

Thus $\operatorname{Pic}^{G}(M)_{0}$ is the connected subgroup of the homologically trivial $G$ linearized line bundles in the sense of $[\mathrm{DH}]$. We shall call the $G$-linearized line bundles in $\operatorname{Pic}^{G}(M)_{0}^{\prime}$ numerically trivial.

Remark 2.1. Clearly $\operatorname{Pic}^{G}(M)_{0} \cong \operatorname{Pic}(M)_{0}$ and $\operatorname{Pic}^{G}(M)_{0}^{\prime} \cong \operatorname{Pic}(M)_{0}^{\prime}$.

Definition 2.2. We shall say that $L_{1}, L_{2} \in \operatorname{Pic}^{G}(M)$ are homologically equivalent, written $L_{1} \sim_{\mathrm{h}} L_{2}$, if $L_{1} \otimes L_{2}^{-1} \in \operatorname{Pic}^{G}(M)_{0}$. We shall say that $L_{1}, L_{2} \in \operatorname{Pic}^{G}(M)$ are numerically equivalent, written $L_{1} \sim_{\mathrm{n}} L_{2}$, if $L_{1} \otimes L_{2}^{-1} \in \operatorname{Pic}^{G}(M)_{0}^{\prime}$.

Let now $\mathrm{NS}^{G}(M)=: \operatorname{Pic}^{G}(M) / \mathrm{Pic}^{G}(M)_{0}$ be the set of all homological equivalence classes (2.3.7 of [DH] $)$. Then $\operatorname{NS}^{G}(M)$ is a finitely generated Abelian group, of rank $\rho^{G}(M)=: \rho(M)+t(\widetilde{G})$, where $\rho(M)$ is the Picard number of $M$, and $t(\widetilde{G})$ is the dimension of the radical of $\widetilde{G}$. Let $\mathrm{NS}^{G}(M)_{\text {tor }} \subseteq$ $\mathrm{NS}^{G}(M)$ be the torsion part of $\mathrm{NS}^{G}(M)$.

Lemma 2.1. $\mathrm{NS}^{G}(M)_{\mathrm{tor}}=\operatorname{Pic}^{G}(M)_{0}^{\prime} / \mathrm{Pic}^{G}(M)_{0}$.

Proof. By (4) and Definition 2.1, we have isomorphisms of Abelian groups

$$
\begin{aligned}
& \operatorname{ker}\left(c_{1}^{(\mathbb{R})} \circ f_{G}\right) \cong \chi(G) \times \operatorname{Pic}(M)_{0}^{\prime} \cong \chi(G) \times \operatorname{Pic}^{G}(M)_{0}^{\prime}, \\
& \operatorname{ker}\left(c_{1}^{(\mathbb{Z})} \circ f_{G}\right) \cong \chi(G) \times \operatorname{Pic}(M)_{0} \cong \chi(G) \times \operatorname{Pic}^{G}(M)_{0} .
\end{aligned}
$$

Under this isomorphism, for any $L \in \operatorname{Pic}(M)_{0}^{\prime}$ its lift $L^{G}$ corresponds to the pair $(1, L)$, and for any integer $m$ the tensor power $\left(L^{G}\right)^{\otimes m}$ corresponds to the pair $\left(1, L^{\otimes m}\right)$. Since $L \in \operatorname{Pic}(M)_{0}^{\prime}, L^{\otimes m} \in \operatorname{Pic}(M)_{0}$, for some integer $m \geq 1$. Therefore, $\left(L^{G}\right)^{\otimes m} \in \operatorname{Pic}^{G}(M)_{0}$, and the homological equivalence class $[L] \in \mathrm{NS}^{G}(M)$ is torsion.

Conversely, suppose $R \in \operatorname{Pic}^{G}(M)$ and that its class $[R]$ in $\operatorname{NS}^{G}(M)$ is torsion. This means that $R^{\otimes m} \in \operatorname{Pic}^{G}(M)_{0}$ for some integer $m \geq 1$. Thus $f_{G}(R)^{\otimes m} \in \operatorname{Pic}(M)_{0}$ and therefore $f_{G}(R) \in \operatorname{Pic}(M)_{0}^{\prime}$. In other words, $R \in \operatorname{ker}\left(c_{1}^{(\mathbb{R})} \circ f_{G}\right) \cong \chi(G) \times \operatorname{Pic}(M)_{0}^{\prime}$.

Suppose then that $R$ corresponds to a pair $(\gamma, L)$, where $\gamma \in \chi(G)$ and $L \in \operatorname{Pic}_{0}(M)^{\prime}$. Then $L^{\otimes m}$ corresponds to $\left(\gamma^{m}, R^{\otimes m}\right)$. In order for this to lie in $\operatorname{Pic}^{G}(M)_{0}$, we need to have $\gamma^{m}=1$, whence $\gamma=1$ (by the connectedness of $G$ ). Thus, $R$ lies in $\operatorname{Pic}^{G}(M)_{0}^{\prime}$. 
Definition 2.3. (2.3.7 of $[\mathrm{DH}]$ ) The G-numerical lattice of $M$ is

$$
\operatorname{Num}^{G}(M)=: \mathrm{NS}^{G}(M) / \mathrm{NS}^{G}(M)_{\text {tor }} .
$$

By Lemma 2.1. $\mathrm{Num}^{G}(M)$ is the set of all numerical equivalence classes:

Corollary 2.1. $\operatorname{Num}^{G}(M) \cong \operatorname{Pic}^{G}(M) / \operatorname{Pic}^{G}(M)_{0}^{\prime}$.

Definition 2.4. The equivariant Néron-Severi space of $M$ is

$$
\mathrm{NS}^{G}(M)_{\mathbb{R}}=: \mathrm{NS}^{G}(M) \otimes_{\mathbb{Z}} \mathbb{R} .
$$

Remark 2.2. $\mathrm{NS}^{G}(M)_{\mathbb{R}}$ is a real vector space of dimension $\rho^{G}(M)$, it contains the numerical lattice and $\mathrm{NS}^{G}(M)_{\mathbb{R}} \cong \operatorname{Num}^{G}(M) \otimes_{\mathbb{Z}} \mathbb{R}$.

Definition 2.5. The $G$-ample cone $\mathrm{C}^{G}(M) \subseteq \mathrm{NS}^{G}(M)_{\mathbb{R}}$ is the convex cone spanned by the classes of all ample $L \in \operatorname{Pic}^{G}(M)$ having non-empty semistable locus.

\subsection{Moment maps}

In the following, by a G-linearized Hermitian line bundle on the projective $G$-variety $M$ we shall mean the choice of $L \in \operatorname{Pic}^{G}(M)$ together with a $G$ invariant Hermitian metric $h$ on $L$. If $M$ is non-singular and $(L, h)$ is a $G$-linearized Hermitian line bundle on $M$, let $X \subseteq L^{*}$ be the corresponding unit circle bundle, and let $\alpha \in \Omega^{1}(X)$ be the connection form for the unique compatible connection. Let $\mathfrak{g}$ be the Lie algebra of $G$. Then we may define an equivariant map $\Phi_{L, h}: M \rightarrow \mathfrak{g}^{*}$ by setting, for every $m \in M$ and $\xi \in \mathfrak{g}$ :

$$
\left\langle\Phi_{L, h}(m), \xi\right\rangle=: \alpha_{x}\left(\xi_{X}(x)\right),
$$

where $x \in X$ is any point lying over $m$, and $\xi_{X}$ is the vector field on $X$ generated by $\xi$ GGK].

Definition 2.6. $\Phi_{L, h}$ will be called a moment map for $L$.

When $L$ is ample, $h$ can be so chosen that the curvature form of the unique compatible connection is $-2 \pi i \Omega$, where $\Omega$ is a $G$-invariant Kähler form. Then the action of $G$ on $M$ is Hamiltonian for $\Omega$, and $\Phi_{L, h}$ is a moment map in the ordinary sense. In particular, since any $L \in \operatorname{Pic}^{G}(M)$ may be factored as $L=A \otimes B^{-1}$, where $A, B \in \operatorname{Pic}^{G}(M)$ are ample, we see that for any $\xi \in \mathfrak{g}$ we have

$$
d \Phi_{L, h}^{\xi}=\iota\left(\xi_{M}\right) \Omega_{L, h},
$$


where $\Phi_{L, h}^{\xi}=:\left\langle\Phi_{L, h}, \xi\right\rangle, \xi_{M}$ denotes the vector field on $M$ generated by $\xi$, and $-2 \pi i \Omega_{L, h}$ is the curvature form of the unique compatible connection. In the following, we shall leave the choice of $h$ implicit, and write $\Phi_{L}$ for $\Phi_{L, h}$.

Up to topological obstructions, the information encoded by $\Phi_{L}$ is equivalent to the assignment of the linearization. More precisely, the infinitesimal action of $\mathfrak{g}$ on the sections of $L$ is given by

$$
\xi \cdot \sigma=: \nabla_{\xi_{M}} \sigma+2 \pi i \Phi_{L}^{\xi} \cdot \sigma \quad\left(\xi \in \mathfrak{g}, \sigma \in \mathcal{C}^{\infty}(M, L)\right),
$$

where $\nabla$ is the associated covariant derivative (equation (5.1) of [GS1]).

Remark 2.3. When $M$ is not necessarily non-singular, we may still define a moment map $\Phi_{L}=\Phi_{L, h}: M \rightarrow \mathfrak{g}^{*}$ by pulling-back $(L, h)$ to some equivariant resolution of singularities $f: \widetilde{M} \rightarrow M[\mathrm{EH}]$, [EV] and checking that $\Phi_{L}$ is well-defined (and smooth) on $M$. Then (71) is satisfied on the smooth locus of $M$. If $L$ is ample, one may equivalently proceed as follows: For every $k \gg 0$, the linear series $\left|L^{\otimes k}\right|$ determines a $G$-equivariant embedding $\varphi_{L^{\otimes k}}: M \rightarrow \mathbb{P} H^{0}\left(M, L^{\otimes k}\right)^{*}$. Let us fix a $G$-invariant Hermitian metric on $H^{0}\left(M, L^{\otimes k}\right)$, so that the linear action of $G$ on $H^{0}\left(M, L^{\otimes k}\right)$ is unitary and the associated action of $G$ on $\mathbb{P} H^{0}\left(M, L^{\otimes k}\right)^{*}$ is Hamiltonian with respect to

the Fubini-Study form $\Omega_{\mathrm{FS}}$. Let $\Phi_{\mathbb{P} H^{0}\left(M, L^{\otimes k}\right)^{*}}: \mathbb{P} H^{0}\left(M, L^{\otimes k}\right)^{*} \rightarrow \mathfrak{g}^{*}$ be the associated moment map, and let us define

$$
\Phi_{L}=: \frac{1}{k} \Phi_{\mathbb{P} H^{0}\left(M, L^{\otimes k}\right)^{*}} \circ \varphi_{L^{\otimes k}}: M \rightarrow \mathfrak{g}^{*}
$$

Again (17) is satisfied on the smooth locus of $M$ when $\Omega=\frac{1}{k} \varphi_{L^{\otimes k}}^{*}\left(\Omega_{\mathrm{FS}}\right)$.

Given (8), the arguments in $\S 5$ and $\S 6$ of GS1 leading to Theorems 5.3 and 6.3 of loc. cit. yield the following

Theorem 2.1. Let $M$ a complex projective $G$-variety, and suppose $L \in$ $\operatorname{Pic}^{G}(M)$. Let $\Phi_{L}: M \rightarrow \mathfrak{g}^{*}$ be a moment map for $L$. Let $\mu$ be a maximal weight for $G$. Suppose that $\mu \notin \Phi_{L}(M) \subseteq \mathfrak{g}^{*}$. Then $H^{0}(M, L)_{\mu}=0$.

We notice in passing the following:

Lemma 2.2. Let $f: N \rightarrow M$ be an equivariant morphism of projective $G$ varieties. Suppose that $L \in \operatorname{Pic}^{G}(M)$ and let $\Phi_{L}: M \rightarrow \mathfrak{g}^{*}$ be a moment map for $L$. Then $\Phi_{L} \circ f: N \rightarrow \mathfrak{g}^{*}$ is a moment map for $f^{*}(L) \in \operatorname{Pic}^{G}(N)$.

\section{$2.3 \quad G$-invariant effectiveness.}

Definition 2.7. Let $M$ a complex projective $G$-variety. 
i): A G-invariantly effective divisor will be an effective divisor $D$ all of whose irreducible components are $G$-invariant; we shall write $D \geq_{G} 0$. In other words, $D \geq_{G} 0$ means that $D=\sum_{i} a_{i} D_{i}$ where $a_{i} \geq 0$ and each $D_{i}$ is irreducible and satisfies $g \cdot D_{i}=D_{i} \forall g \in G$.

ii): A $G$-invariantly effective line bundle on $M$ is an $L \in \operatorname{Pic}^{G}(M)$ possessing a non-zero $G$-invariant section $0 \neq \sigma \in H^{0}(M, L)^{G}$.

Remark 2.4. The notion of $G$-invariant effectiveness for $G$-linearized line bundles differs from the notion of $G$-effectiveness introduced in [DH] (which means that $\left.M^{\mathrm{ss}}(L) \neq \emptyset\right)$.

Remark 2.5. If $L \in \operatorname{Pic}^{G}(M)$ is $G$-invariantly effective and $0 \neq \sigma \in H^{0}(M, L)^{G}$, then $L=\mathcal{O}_{M}\left(Z_{\sigma}\right)$ for a $G$-invariantly effective Cartier divisor $Z_{\sigma}=\operatorname{zero}(\sigma) \geq_{G}$ 0 , but the converse needn't be true. Some power $L^{\otimes k}, k \geq 1$, is $G$-invariantly effective if and only if $M^{\mathrm{ss}}(L) \neq \emptyset$ (that is, if and only if $L$ is $G$-effective in the sense of $[\mathrm{DH}]$ ).

Remark 2.6. Suppose that $L \in \operatorname{Pic}^{G}(M)$ is $G$-invariantly effective, and pick a non-zero $\sigma \in H^{0}(M, L)^{G}$. Given any $H \in \operatorname{Pic}^{G}(M)$, tensor product by $\sigma^{\otimes k}$ defines a $G$-equivariant injective morphism of line bundles $H^{\otimes k} \rightarrow(H \otimes$ $L)^{\otimes k}$. Thus for every maximal weight there is an induced injective linear map $H^{0}\left(M, H^{\otimes k}\right)_{\mu} \rightarrow H^{0}\left(M,(H \otimes L)^{\otimes k}\right)_{\mu}$. Passing to lim sup, we obtain

$$
\operatorname{vol}_{\mu}(H) \leq \operatorname{vol}_{\mu}(H \otimes L)
$$

for every maximal weight $\mu$.

\subsection{Regular $G$-linearized line bundles}

Definition 2.8. Suppose that $B \in \operatorname{Pic}^{G}(M)$ is ample. We shall say that $B$ is regular if its stable and semi-stable loci coincide, and are non-empty: $M^{\mathrm{s}}(B)=M^{\mathrm{ss}}(B) \neq \emptyset$.

Remark 2.7. Let $\mathrm{NS}^{G}(M)_{\mathbb{R}}$ be the equivariant real Néron-Severi space of $M$, and let $\mathrm{C}^{G}(M) \subseteq \mathrm{NS}^{G}(M)_{\mathbb{R}}$ be the $G$-ample cone $[\mathrm{DH}]$. Then $B \in \operatorname{Pic}^{G}(M)$ is regular if and only if its numerical equivalence class $[B] \in \mathrm{NS}^{G}(M)_{\mathbb{R}}$ lies in the interior of some open chamber of $\mathrm{C}^{G}(M)$.

Remark 2.8. Simplectically, regularity may be formulated as follows: Let $\Phi_{B}: M \rightarrow \mathfrak{g}^{*}$ be a moment map associated to the $G$-line bundle $B$. Then $B$ is regular if and only if $0 \in \mathfrak{g}^{*}$ is a regular value of $\Phi_{B}$, and $\Phi_{B}^{-1}(0) \neq \emptyset[\mathrm{Ki1}$. 
Remark 2.9. If $B \in \mathrm{Pic}^{G}(M)$ is ample and regular, the GIT quotient $M_{0}(B)$ with respect to $B$ is a complex orbifold, and $B$ descends to a line orbibundle $B_{0}$ on $M_{0}(B)$. If $\Omega_{B}$ is a $G$-invariant Kähler form representing $c_{1}(B)$, it descends to a Kähler form $\Omega_{0}$ on $M_{0}(B)$ representing $c_{1}\left(B_{0}\right)$.

Definition 2.9. Let $M$ be a complex projective $G$-manifold, and suppose $L \in \operatorname{Pic}^{G}(M)$ is ample and has non-empty stable locus: $M^{\mathrm{s}}(L) \neq \emptyset$. Then there exist a $G$-equivariant birational morphism $f: \widetilde{M} \rightarrow M$ from a complex projective $G$-manifold $\widetilde{M}$, and an effective $G$-invariant exceptional divisor $E \subseteq \widetilde{M}$, such that $f^{*}(L)^{\otimes k}(-E) \in \operatorname{Pic}^{G}(\widetilde{M})$ is ample and regular for every $k \gg 0$ Ki2 . Furthermore, $f$ is an isomorphism over the stable points of $L$. We shall refer to a morphism with these properties as a Kirwan resolution of $(M, L)$.

Remark 2.10. The $G$-action on $\mathcal{O}_{\widetilde{M}}(E)$ constructed in $\S 3$ of [Ki2] is such that $\mathcal{O}_{\tilde{M}}(E)$ is $G$-invariantly effective, i.e. the natural morphism (well-defined up to a non-zero scalar factor) $\mathcal{O}_{\widetilde{M}} \rightarrow \mathcal{O}_{\widetilde{M}}(E)$ is $G$-equivariant (the action of $G$ on the restriction of $\mathcal{O}_{\widetilde{M}}(E)$ to $\widetilde{M} \backslash E$ is the product of the action of $G$ on $\widetilde{M} \backslash E$ with the trivial action on $\mathbb{C}$ ).

\subsection{Associated characters.}

Let us first recall some results and terminology from Appendix B of [GGK]:

Definition 2.10. Suppose that $M$ is a complex projective $G$-manifold. Let $\operatorname{Conj}(G)_{\text {subgr }}$ be the collection of all conjugacy classes of subgroups of $G$ (that is, an element of $\operatorname{Conj}(G)_{\text {subgr }}$ is the collection of all subgroups of $G$ conjugate to a given one). There exists a subgroup $K \subseteq G$ such that the stabilizer of a general $p \in M$ is conjugate to $K$. Then its conjugacy class $(K) \in \operatorname{Conj}(G)_{\text {subgr }}$ is called the principal orbit type of the action of $G$ on $M$.

Remark 2.11. Suppose that $M$ is a complex projective $G$-manifold, with principal orbit type $(K)$. If there exists an ample $L \in \operatorname{Pic}^{G}(M)$ with nonempty stable locus, then $K \subseteq G$ is a finite subgroup.

Let us momentarily suppose that the stabilizer of a general $p \in M$ is a central subgroup, so that the principal orbit type of the action consists of a single subgroup $K \subseteq G$.

Definition 2.11. For every $L \in \operatorname{Pic}^{G}(M)$, let us denote by $\chi_{K, L}: K \rightarrow S^{1}$ the character corresponding to the linear action of $K$ on the fiber of $L$ at a general $p \in M$. 
Remark 2.12. Let $K^{*}=: \operatorname{Hom}\left(K, S^{1}\right)$. Then $\chi: L \in \operatorname{Pic}^{G}(M) \mapsto \chi_{K, L} \in K^{*}$ is a group homomorphism. Obviously, $\operatorname{ker}(\chi)$ contains every $G$-invariantly effective $L \in \operatorname{Pic}^{G}(M)$. In particular, if $L, L^{\prime} \in \operatorname{Pic}^{G}(M)$, and there exists a $G$-equivariant morphism $L \rightarrow L^{\prime}$, then $\chi_{K, L}=\chi_{K, L^{\prime}}$. In the situation of Remark 2.10, one has $\chi_{K, \mathcal{O}_{\tilde{M}}(E)}=1$.

Remark 2.13. If $K$ is not necessarily central, we can still define $\chi_{K_{p}, L}: K_{p} \rightarrow$ $S^{1}$ for the general $p \in M\left(K_{p}\right.$ is the stabilizer of $\left.p\right)$. The condition $\chi_{K_{p}, L}=1$ is independent of $p$.

Definition 2.12. Suppose again that the stabilizer of the general $p \in M$ is a central subgroup $K \subseteq G$. Choose a dominant weight $\mu$. If $T \subseteq G$ is the chosen maximal torus, let $\mu_{T}: T \rightarrow S^{1}$ be the character induced by $\mu$ by exponentiation, $\mu_{T}: \exp _{T}(\xi) \mapsto e^{2 \pi i<\mu, \xi>}$. Given that $K \subseteq T$, we may define $\mu_{K}=:\left.\mu_{T}\right|_{K}: K \rightarrow S^{1}$.

Remark 2.14. An alternative description of $\mu_{K}$ is as follows: Let $\mathcal{O}_{\mu} \subseteq \mathfrak{g}^{*}$ be the coadjoint orbit of $\mu$. Since $\mu$ is an integral weight, the natural Kähler structure on $\mathcal{O}_{\mu}$ is in fact a Hodge form, that is, it represents an integral cohomology class. The associated ample holomorphic line bundle $A_{\mu} \rightarrow \mathcal{O}_{\mu}$ is a $G$-line bundle in a natural manner. Since the action of $K$ is trivial on $\mathcal{O}_{\mu}$, as in Definition 2.11 the linearization induces the character $\mu_{K}$ on $K$.

Definition 2.13. In the hypothesis and notation of Definitions 2.11 and 2.12 we shall say that $L \in \operatorname{Pic}^{G}(M)$ and a given dominant weight $\mu$ are numerically compatible if for some $r \in\{1, \ldots,|K|\}$ we have $\chi_{K, L}^{r} \cdot \bar{\mu}_{K}=1$ (the constant character equal to 1 ).

Obviously, any $L \in \operatorname{Pic}^{G}(M)$ is numerically compatible with $\mu=0$ (corresponding to the trivial representation).

\subsection{Equivariant exponents}

Definition 2.14. If $L \in \operatorname{Pic}^{G}(M)$, the $G$-semigroup of $G$ is

$$
\mathbb{N}_{G}(L)=:\left\{m \geq 0: H^{0}\left(M, L^{\otimes m}\right)^{G} \neq\{0\}\right\} .
$$

Assuming $\mathbb{N}_{G}(L) \neq\{0\}$, the $G$-exponent of $L$ is the greatest common divisor of the elements of $\mathbb{N}_{G}(L)$. Thus $e_{G}(L)$ divides every element of $\mathbb{N}_{G}(L)$, and every sufficiently large integral multiple of $e_{G}(L)$ belongs to $\mathbb{N}_{G}(L)$.

For every maximal weight $\mu$, we shall let

$$
\mathbb{N}_{\mu}(L)=:\left\{m \in \mathbb{N}: H^{0}\left(M, L^{\otimes m}\right)_{\mu} \neq\{0\}\right\}
$$


The semigroup $\mathbb{N}_{G}(L)$ acts on $\mathbb{N}_{\mu}(L)$ by translations.

Example 2.1. The following is a consequence of Theorem 3.1 below. Suppose that the stabilizer $K \subseteq G$ of a general $p \in M$ is a central subgroup, and that $L \in \operatorname{Pic}^{G}(M)$ is ample and regular. Let $\chi_{K, L}: K \rightarrow S^{1}$ be the character associated to the linearization $L$. Then $e_{G}(L)$ is the period of $\chi_{K, L}$. If $\mu$ is a maximal weight, it determines a character $\mu_{K}: K \rightarrow S^{1}$. Then $\mathbb{N}_{\mu}(L) \neq\{0\}$ if and only if there exists $r \in \mathbb{N}$ such that $\chi_{K, L}^{r} \cdot \bar{\mu}_{K}=1$. In this case, if $k \gg 0$ then $k \in \mathbb{N}_{\mu}(L)$ if and only if $k \in r+\mathbb{N}_{G}(L)$. Thus, up to a finite number of terms, $\mathbb{N}_{G}(L)$ consists of all multiples of the period of $\chi_{K, L}$, and $\mathbb{N}_{\mu}(L)$ consists of their translates by $r$.

Lemma 2.3. For any $L \in \operatorname{Pic}^{G}(M)$ and any $p \in \mathbb{N}$, we have

$$
e_{G}\left(L^{\otimes p}\right)=\frac{e_{G}(L)}{\operatorname{gcd}\left(p, e_{G}(L)\right)}
$$

Example 2.2. Referring to the situation of Example [2.1, $e_{G}\left(L^{\otimes p}\right)$ is the period of $\chi_{K, L}^{\otimes p},\left|\chi_{K, L}^{\otimes p}\right|$. Thus,

$$
e_{G}\left(L^{\otimes p}\right)=\left|\chi_{K, L} \otimes p\right|=\left|\left(\chi_{K, L}\right)^{p}\right|=\frac{\left|\chi_{K, L}\right|}{\operatorname{gcd}\left(p,\left|\chi_{K, L}\right|\right)}=\frac{e_{G}(L)}{\operatorname{gcd}\left(p, e_{G}(L)\right)} .
$$

Proof of Lemma 2.3. Let us set $e^{\prime}=: e_{G}(L) / \operatorname{gcd}\left(p, e_{G}(L)\right)$; hence $e_{G}(L)=$ $e^{\prime} \cdot \operatorname{gcd}\left(p, e_{G}(L)\right), p=p^{\prime} \cdot \operatorname{gcd}\left(p, e_{G}(L)\right)$ where $e^{\prime}$ and $p^{\prime}$ are relatively prime. By definition, $H^{0}\left(M, L^{\otimes p e_{G}\left(L^{\otimes p}\right) m}\right)^{G} \neq\{0\}$ for every $m \gg 0$. Thus,

$$
e_{G}(L)\left|p e_{G}\left(L^{\otimes p}\right) \Rightarrow e^{\prime}\right| p^{\prime} e_{G}\left(L^{\otimes p}\right) \Rightarrow e^{\prime} \mid e_{G}\left(L^{\otimes p}\right) \text {. }
$$

On the other hand, since $p e^{\prime}=e_{G}(L)\left(p / \operatorname{gcd}\left(p, e_{G}(L)\right)\right.$ is an integral multiple of $e_{G}(L)$, we have $H^{0}\left(M, L^{\otimes p e^{\prime} m}\right)^{G} \neq\{0\}$ for every $m \gg 0$. Therefore, $e_{G}\left(L^{\otimes p}\right) \mid e^{\prime}$.

\section{Equivariant volumes and GIT quotients}

In the action-free case, the volume of a nef and big line bundle is computed by the top self-intersection of the first Chern class. With this in mind, we shall now examine some special cases where the equivariant volumes admit a similar interpretation, in terms of suitable top self-intersections on appropriate GIT quotients of $M$.

For simplicity and ease of exposition, we shall focus on the special case where the stabilizer $K \subseteq G$ of a general $p \in \Phi^{-1}(0)$ is a central subgroup 
$K \subseteq G$, which in the hypothesis of the present section is necessarily finite. In view of coisotropic embedding Theorem GS3, $K$ is then the stabilizer of a general $p \in M$. The proofs below can however be extended with no conceptual difficulty to the case of an arbitrary principal orbit type. This will involve singling out for each maximal weight $\mu$ the kernel $K_{\mu} \subseteq K$ of the action of $K$ on the coadjoint orbit of $\mu$, and considering the contribution coming from each conjugacy class of $K_{\mu}$.

The first case that we consider is the one of ample and regular $G$-linearized line bundles (Definition 2.8).

Let us recall that if $L \in \mathrm{Pic}^{G}(M)$ is ample and regular, then the GIT quotient of $M$ with respect to $L, M_{0}(L)$, may be described as a symplectic reduction with respect to a $G$-invariant Kähler form $\Omega$ representing $c_{1}(L)$. More precisely, recall that by Remark 2.8 $0 \in \mathfrak{g}^{*}$ is a regular value of $\Phi$, and $\Phi^{-1}(0) \neq \emptyset$. Furthermore, $P=: \Phi^{-1}(0)$ is a connected $G$-invariant codimension $g$ submanifold of $M$, on which $G$ acts locally freely. There is a natural identification between the GIT quotient $M_{0}(L)=M^{s}(L) / \tilde{G}$ and the symplectic reduction $P / G$, which carries an induced structure of Kähler orbifold. In this manner, $\Omega$ descends to a Kähler form (in the orbifold sense) $\Omega_{0}$ on $M_{0}(L)$, representing $c_{1}\left(L_{0}\right)$ - here $L_{0}$ is orbifold line bundle that $L$ descends to on $M_{0}(L)$.

Theorem 3.1. Let $M$ be a complex projective manifold, $G$ a compact connected Lie group, $\nu: G \times M \rightarrow M$ a holomorphic action. Suppose (for simplicity) that the stabilizer of a general $p \in \Phi^{-1}(0)$ is a central subgroup $K \subseteq G$. Let $L \in \operatorname{Pic}^{G}(M)$ be ample and regular, and let $\Phi=\Phi_{L}: M \rightarrow \mathfrak{g}^{*}$ be a moment map for $L$, associated to the Kähler form $\Omega$ representing $c_{1}(L)$. Let $M_{0}(L)=: \Phi^{-1}(0) / G$ be the symplectic quotient with respect to $\Phi_{L}$. Let $\mu \in \Lambda_{+}$be a dominant weight. If $L$ and $\mu$ are not numerically compatible (Definition 2.13), then $H_{\mu}^{0}\left(M, L^{\otimes k}\right)=0$ for every $k=1,2, \ldots$ If on the other hand $L$ and $\mu$ are numerically compatible, then

$$
\operatorname{vol}_{\mu}(L)=\operatorname{dim}\left(V_{\mu}\right)^{2} \cdot \operatorname{vol}\left(M_{0}(L), \Omega_{0}\right)>0 .
$$

Here

$$
\operatorname{vol}\left(M_{0}(L), \Omega_{0}\right)=: \int_{M_{0}(L)} \Omega_{0}^{\wedge(n-g)},
$$

The quickest way to prove this is by applying the Riemann-Roch formulae for multiplicities due to Meinrenken [M1, [M2]. A fairly elementary analytic approach, based on the microlocal theory of the Szegö kernel from [BS] and $[\mathrm{Z}$, has been used in [P2] in the case of generically free actions.

Proof. For $k \geq 1$, let us replace $L$ by $L^{\otimes k}$, the Hodge form $\Omega$ and the moment map $\Phi$ by their multiples $k \Omega$ and $\Phi_{k}=: k \Phi$. Given any $\mu \in$ 
$\mathfrak{g}^{*}$, there exists $k_{0}$ such that $\mu$ is a regular value of $\Phi_{k}$ if $k \geq k_{0}$. The relevant asymptotic information about the multiplicity of $V_{\mu}$ in $H^{0}\left(M, L^{\otimes k}\right)$ may then determined by computing appropriate Riemann-Roch numbers on the orbifold obtained as symplectic reduction at the coadjoint orbit $\mathcal{O}_{\mu} \subseteq \mathfrak{g}^{*}$ of $\mu$ [Ka], $\mathrm{M} 2$.

More precisely, since $\mu$ is integral the Kirillov symplectic form $\sigma_{\mu}$ on $\mathcal{O}_{\mu}$ is a Hodge form. By the Kostant version of the Borel-Bott theorem, there is an ample line bundle $A_{\mu}$ on $\mathcal{O}_{\mu}$ such that $H^{0}\left(\mathcal{O}_{\mu}, A_{\mu}\right)$ is the irreducible representation of $G$ with highest weight $\mu$.

Let $M_{\mu}^{(k)}$ be the Weinstein symplectic reduction of $M$ at $\mu$ with respect to the moment map $\Phi_{k}=k \Phi_{L}(k \gg 0)$. Using the normal form description of the symplectic and Hamiltonian structure of $(M, \Omega)$ in the neighbourhood of the coisotropic submanifold $\left.P=\Phi^{-1}(0)[\mathrm{M} 2], \mathrm{G}\right]$, GS3], one can verify that $M_{\mu}^{(k)}$ is, up to diffeomorphism, the quotient of $P \times \mathcal{O}_{\mu}$ by the product action of $G$. In other words, $M_{\mu}^{(k)}$ is the fibre orbibundle on $M_{0}=: P / G$ associated to the principal $G$-orbibundle $q: P \rightarrow M_{0}$ and the $G$-space $\mathcal{O}_{\mu}$ (endowed with the opposite Kähler structure); in particular its diffeotype is independent of $k$ for $k \gg 0$. Let $p_{\mu}: M_{\mu}^{(k)} \rightarrow M_{0}$ be the projection.

Let $\theta$ be a connection 1-form for $q$ (GGK], Appendix B). By the shifting trick, the symplectic structure $\Omega_{\mu}^{(k)}$ of the orbifold $M_{\mu}^{(k)}$ is obtained by descending the closed 2-form $k \iota^{*}(\Omega)+<\mu, F(\theta)>-\sigma_{\mu}$ on $P \times \mathcal{O}_{\mu}$ down to the quotient (the symbols of projections are omitted for notational simplicity). The minimal coupling term $\left\langle\mu, F(\theta)>-\sigma_{\mu}\right.$ is the curvature of the line orbibundle $R_{\mu}=\left(P \times \bar{A}_{\mu}\right) / G$ on $M_{\mu}^{(k)}$. Thus, $\Omega_{\mu}^{(k)}$ is the curvature form of the line orbibundle $p_{\mu}^{*}\left(L_{0}^{\otimes k}\right) \otimes R_{\mu}$.

Let

$$
\begin{aligned}
\widetilde{P}_{\mu}=: & \left\{\left(p, \mu^{\prime}, g\right) \in P \times \mathcal{O}_{\mu} \times G: g \cdot\left(p, \mu^{\prime}\right)=\left(p, \mu^{\prime}\right)\right\}, \\
\widetilde{P}_{\mu, K}=: & P \times \mathcal{O}_{\mu} \times K .
\end{aligned}
$$

Let $G$ act on itself by $h \cdot g=: h g h^{-1}$, and on $\widetilde{P}_{\mu}$ by the product action. Since $K$ acts trivially on $P$ and (being central) on $\mathcal{O}_{\mu}$, there is a natural inclusion $\widetilde{P}_{\mu, K} \subseteq \widetilde{P}_{\mu}$. Now let $\Sigma_{\mu}=: \tilde{P}_{\mu} / G, \Sigma_{\mu, K}=: \tilde{P}_{\mu, K} / G=M_{\mu}^{(k)} \times K$. There is a natural orbifold complex immersion $\Sigma_{\mu} \rightarrow M_{\mu}^{(k)}$, with complex normal orbi-bundle $N_{\Sigma_{\mu}}$, and $\Sigma_{\mu, K} \subseteq \Sigma_{\mu}$ is the union of the $|K|$ connected components mapping dominantly (and isomorphically) onto $M_{\mu}^{(k)}$. The orbifold multiplicity of $\Sigma_{\mu, K}$ is constant and equal to $|K|$. Let $L_{0}$ be the line orbibundle on $M_{0}$ determined by descending $L$, and let $\tilde{L}_{0}$ be its pull-back to $\Sigma_{\mu}$. Let $\mathrm{r}$ be the complex dimension of $\mathcal{O}_{\mu}$, so that $\operatorname{dim} M_{\mu}^{(k)}=\mathrm{n}-\mathrm{g}+\mathrm{r}$. After [M1] and [M2], the multiplicity $N^{(k)}(\mu)$ of the irreducible representation $V_{\mu}$ 
in $H^{0}\left(M, L^{\otimes k}\right)$ is then given by:

$$
\begin{aligned}
N^{(k)}(\mu)= & \int_{\Sigma_{\mu}} \frac{1}{d_{\Sigma_{\mu}}} \frac{\operatorname{Td}\left(\Sigma_{\mu}\right) \mathrm{Ch}^{\Sigma_{\mu}}\left(p_{\mu}^{*}\left(L_{0}^{\otimes k}\right) \otimes R_{\mu}\right)}{\mathrm{D}^{\Sigma_{\mu}}\left(N_{\Sigma_{\mu}}\right)} \\
= & \int_{\Sigma_{\mu, K}} \frac{1}{d_{\Sigma_{\mu}}} \frac{\operatorname{Td}\left(\Sigma_{\mu}\right) \mathrm{Ch}^{\Sigma_{\mu}}\left(p_{\mu}^{*}\left(L_{0}^{\otimes k}\right) \otimes R_{\mu}\right)}{\mathrm{D}^{\Sigma_{\mu}}\left(N_{\Sigma_{\mu}}\right)}+O\left(k^{\mathrm{n}-\mathrm{g}-1}\right) \\
= & k^{\mathrm{n}-\mathrm{g}} \frac{1}{|K|} \sum_{h \in K} \chi_{K, L}(h)^{k} \overline{\mu_{K}(h)} \int_{M_{\mu}^{(k)}} \frac{\left(k c_{1}\left(L_{0}\right)+c_{1}\left(R_{\mu}\right)\right)^{\mathrm{n}-\mathrm{g}+\mathrm{r}}}{(\mathrm{n}-\mathrm{g}+\mathrm{r}) !} \\
& +O\left(k^{\mathrm{n}-\mathrm{g}-1) .}\right.
\end{aligned}
$$

Now suppose that $\chi_{K, L}^{k} \cdot \overline{\mu_{K}} \not \equiv 1$. Then the action of $K$ on $\jmath^{*}\left(L^{\otimes k}\right) \otimes \bar{A}_{\mu}$ is not trivial, where $\jmath: P \hookrightarrow M$ is the inclusion. Therefore, the fiber of $p_{\mu}^{*}\left(L_{0}^{\otimes k}\right) \otimes R_{\mu}$ on the smooth locus of $M_{0}$ is a nontrivial quotient of $\mathbb{C}$, and $N^{(k)}(\mu)=0$ in this case. If there exists $k$ such that $\chi_{K, L}^{k} \cdot \overline{\mu_{K}} \equiv 1$, on the other hand, the same condition holds with $k$ replaced by $k+\ell e$, where $e$ is the period of $\chi_{K, L}$ and $\ell \in \mathbb{Z}$ is arbitrary. Thus $k$ may be assumed arbitrarily large. Passing to the original Kähler structure of $\mathcal{O}_{\mu}$ in the computation, and recalling that $\operatorname{dim}\left(V_{\mu}\right)=(r !)^{-1} \int_{\mathcal{O}_{\mu}} \sigma_{\mu}^{r}$, we easily obtain:

$$
N^{(k)}(\mu)=\operatorname{dim}\left(V_{\mu}\right) \frac{k^{\mathrm{n}-\mathrm{g}}}{(\mathrm{n}-\mathrm{g}) !} \int_{M_{0}} c_{1}\left(L_{0}\right)^{\wedge(\mathrm{n}-\mathrm{g})}+O\left(k^{\mathrm{n}-\mathrm{g}-1}\right) .
$$

As a Corollary of the proof, we obtain:

Corollary 3.1. In the situation of Theorem 3.1, let $e=e_{L}$ be the period of $\chi_{K, L}$. Then for every $l \gg 0$ we have

$$
\operatorname{dim} H^{0}\left(M, L^{\otimes e l}\right)^{G}=O\left(l^{\mathrm{n}-\mathrm{g}}\right) .
$$

Let us illustrate Theorem 3.1 by a few examples.

Example 3.1. Consider the linear action $S^{1} \times \mathbb{C}^{2} \rightarrow \mathbb{C}^{2}$ given by $t \cdot\left(z_{0}, z_{1}\right)=$ $\left(t z_{0}, t^{-1} z_{1}\right)$. This descends to an action on $\mathbb{P}^{1}$ with an obvious linearization to the hyperplane bundle $\mathcal{O}_{\mathbb{P}^{1}}(1)$. One easily checks that $\operatorname{vol}_{\mu}\left(\mathcal{O}_{\mathbb{P}^{1}}(1)\right)=1$ for every $\mu \in \mathbb{Z}$, but $\operatorname{vol}_{\mu}\left(\mathcal{O}_{\mathbb{P}^{1}}(2)\right)=0$ whenever $\mu$ is odd. To see that this agrees with Theorem 3.1. let us remark that $K=\{ \pm 1\}$, that $\chi_{K, \mathcal{O}_{\mathbb{P} 1}(1)}: K \rightarrow \mathbb{C}^{*}$ is the inclusion, and that for every $\mu \in \mathbb{Z}$ the character $\mu_{K}: K \rightarrow \mathbb{C}^{*}$ is exponentiation by $\mu$. It follows that $\chi_{K, \mathcal{O}_{\mathbb{P} 1}(1)} \cdot \bar{\mu}_{K}=1$ if $\mu$ is odd, and $\chi_{K, \mathcal{O}_{\mathbb{P} 1}(1)}^{2} \cdot \bar{\mu}_{K}=1$ if $\mu$ is even. On the other hand, $\chi_{K, \mathcal{O}_{\mathbb{P} 1}(2)}=\chi_{K, \mathcal{O}_{\mathbb{P} 1}(1)}^{2}=1$, and therefore there does not exist $r \in \mathbb{N}$ such that $\chi_{K, \mathcal{O}_{\mathbb{P} 1}(2)}^{r} \cdot \bar{\mu}_{K}=1$ when $\mu$ is odd. 
Example 3.2. For an example with a positive-dimensional quotient, let us now start from the linear action $S^{1} \times \mathbb{C}^{3} \rightarrow \mathbb{C}^{3}$ given by $t \cdot\left(z_{0}, z_{1}, z_{2}\right)=$ : $\left(t^{-1} z_{0}, t z_{1}, t z_{2}\right)$. Then with the induced linearization $\mathcal{O}_{\mathbb{P}^{2}}(1)$ is a regular ample $S^{1}$-linearized line bundle on $\mathbb{P}^{2}$, with associated moment map

$$
\Phi\left(\left[z_{0}: z_{1}: z_{2}\right]\right)=\frac{\left|z_{1}\right|^{2}+\left|z_{2}\right|^{2}-\left|z_{0}\right|^{2}}{\|z\|^{2}} .
$$

The map $\left[z_{0}: z_{1}: z_{2}\right] \in \Phi^{-1}(0) \mapsto\left[z_{1}: z_{2}\right] \in \mathbb{P}^{1}$ is well-defined and $S^{1}$ invariant, and shows that $\mathbb{P}^{2} / / \mathbb{C}^{*}=\Phi^{-1}(0) / S^{1}=\mathbb{P}^{1}$. Furthermore, $\mathcal{O}_{\mathbb{P}^{2}}(1)$ does not descend to a genuine line bundle on $\mathbb{P}^{1}$, but so does $\mathcal{O}_{\mathbb{P}^{2}}(2)$. For every $k=0,1,2, \ldots$ and $\mu=0,1,2, \ldots$ we have

$H^{0}\left(\mathbb{P}^{2}, \mathcal{O}_{\mathbb{P}^{2}}(k)\right)_{\mu}=\left\{\begin{array}{ccc}\operatorname{span}\left\{z_{0}^{k-a-b} z_{1}^{a} z_{2}^{b}: a+b=\frac{k+\mu}{2}\right\} & \text { if } & 2 \mid k-\mu \geq 0 \\ 0 & \text { otherwise. }\end{array}\right.$

It follows first that setting $\mu=0, k=2 r$ we obtain

$$
\begin{aligned}
\operatorname{dim} H^{0}\left(\mathbb{P}^{2}, \mathcal{O}_{\mathbb{P}^{2}}(2 r)\right)_{0} & =\operatorname{dim} H^{0}\left(\mathbb{P}^{2}, \mathcal{O}_{\mathbb{P}^{2}}(2 r)\right)^{G}=\operatorname{dim} \mathbb{C}\left[z_{1}, z_{2}\right]_{r} \\
& =1+r=\operatorname{dim} H^{0}\left(\mathbb{P}^{1}, \mathcal{O}_{\mathbb{P}^{1}}(r)\right) .
\end{aligned}
$$

This implies that $\mathcal{O}_{\mathbb{P}^{2}}(2)$ descends to the hyperplane line bundle on $\mathbb{P}^{1}$, and therefore if we descend the Fubini-Study form on $\mathbb{P}^{2}$ to a Kähler form $\Omega_{0}$ on $\mathbb{P}^{1}$, the latter satisfies $\operatorname{vol}\left(\mathbb{P}^{1}, \Omega_{0}\right)=\int_{\mathbb{P}^{1}} \Omega_{0}=\frac{1}{2}$.

Next, if in general $k=\mu+2 r, r \geq 0$, then

$$
\begin{aligned}
\operatorname{vol}_{\mu}\left(\mathcal{O}_{\mathbb{P}^{2}}(1)\right) & =\limsup _{r \rightarrow+\infty} \frac{1}{\mu+2 r} \operatorname{dim} H^{0}\left(\mathbb{P}^{2}, \mathcal{O}_{\mathbb{P}^{2}}(k)\right)_{\mu} \\
& =\limsup _{r \rightarrow+\infty} \frac{1}{\mu+2 r} \operatorname{dim} \mathbb{C}\left[z_{1}, z_{2}\right]_{\mu+r}=\frac{1}{2}
\end{aligned}
$$

Example 3.3. For an non-abelian example, let us consider the injective group homomorphism $\alpha: \operatorname{SL}(2) \rightarrow \mathrm{SL}(4)$ given by

$$
\alpha(A)=\left(\begin{array}{cc}
A & 0 \\
0 & \left(A^{t}\right)^{-1}
\end{array}\right) .
$$

This defines a linear action of $\mathrm{SL}(2)$ on $\mathbb{C}^{4}$, whence an action on $\mathbb{P}^{3}$ with a built-in linearization to the hyperplane line bundle. Let us restrict this to the maximal compact subgroup $\mathrm{SU}(2) \subseteq \mathrm{SL}(2)$, and identify $\mathfrak{s u}(2)^{*} \cong \mathfrak{s u}(2)$ by means of the Hermitian form $(A, B) \mapsto \operatorname{trace}\left(A \bar{B}^{t}\right)$. The moment map $\Phi: \mathbb{P}^{3} \rightarrow \mathfrak{s u}(2) \cong \mathbb{R} \oplus \mathbb{C}$ is given by

$$
\Phi\left(\left[z_{0}: z_{1}: z_{2}: z_{3}\right]\right)=\frac{1}{\|z\|^{2}}\left(\begin{array}{c}
\left|z_{0}\right|^{2}-\left|z_{1}\right|^{2}+\left|z_{2}\right|^{2}-\left|z_{3}\right|^{2} \\
z_{1} \overline{z_{0}}+z_{2} \overline{z_{3}}
\end{array}\right) .
$$


Arguing in affine coordinates, one can see that $0 \in \mathfrak{s u}(2)$ is a regular value of $\Phi$, and clearly $\Phi^{-1}(0) \neq \emptyset$. Thus, $\mathcal{O}_{\mathbb{P}^{3}}(1)$ is a regular $\mathrm{SU}(2)$-linearized line bundle on $\mathbb{P}^{3}$. Let us set $V=: \mathbb{C}^{2}, W=: \mathbb{C}^{4}$. There is an isomorphism of $\mathrm{SU}(2)$-modules $W \cong V \oplus V^{*} \cong V \oplus \bar{V} \cong V \oplus V$. Therefore, as $\mathrm{SU}(2)$-modules,

$$
\begin{aligned}
H^{0}\left(\mathbb{P}^{3}, \mathcal{O}_{\mathbb{P}^{3}}(k)\right) & \cong \operatorname{Sym}^{k}(V \oplus V) \cong \bigoplus_{a=0}^{k} \operatorname{Sym}^{a}(V) \otimes \operatorname{Sym}^{k-a}(V) \\
& \cong \bigoplus_{a=0}^{k} \operatorname{Sym}^{k}(V) \oplus \operatorname{Sym}^{k-2}(V) \oplus \cdots \oplus \operatorname{Sym}^{|k-2 a|}(V) \\
& \cong \bigoplus_{l=0}^{\left[\frac{k}{2}\right]} \operatorname{Sym}^{k-2 l}(V)^{\oplus(k-2 l+1)}
\end{aligned}
$$

It follows from (12) that for every $\mu=0,1,2, \ldots$ we have

$$
H^{0}\left(\mathbb{P}^{3}, \mathcal{O}_{\mathbb{P}^{3}}(k)\right)_{\mu}=\left\{\begin{array}{ccc}
\operatorname{Sym}^{\mu}(V)^{\oplus(\mu+1)} & \text { if } & \mu \leq k, 2 \mid k-\mu \\
0 & \text { otherwise. }
\end{array}\right.
$$

We see from (13) that $\operatorname{dim} H^{0}\left(\mathbb{P}^{3}, \mathcal{O}_{\mathbb{P}^{3}}(k)\right)_{\mu}=(\mu+1)^{2}$ if $0 \leq \mu \leq k, 2 \mid k-\mu$. It follows that $\operatorname{vol}_{\mu}\left(\mathcal{O}_{\mathbb{P}^{3}}(1)\right)=\left(\operatorname{dim} V_{\mu}\right)^{2}$ for every $\mu=0,1,2, \ldots$ To see that this agrees with Theorem [3.1, let us remark that $K=\left\{ \pm \mathrm{id}_{2}\right\}$ and that $\chi_{K, \mathcal{O}_{\mathbb{P} 3}(1)}\left( \pm \mathrm{id}_{2}\right)= \pm 1$. On the other hand, for every $\mu=0,1,2, \ldots$ the character $\mu_{K}$ is given by $\mu_{K}\left( \pm \mathrm{id}_{2}\right)=( \pm 1)^{\mu}$.

For future reference, and referring now to the general case (that is, when the stabilizer of a general $p \in M$ is not necessarily a central subgroup of $G$ ), we shall next record a Corollary of the proof of Theorem 3.1 .

Remark 3.1. In the situation of Definition 2.10, let us suppose that there exists $L \in \operatorname{Pic}^{G}(M)$ ample and regular (or simply with non-empty stable locus). Then any subgroup $K \subseteq G$ representing the principal orbit type of the action is finite. If in addition $L$ is $G$-invariantly effective, that is, $\operatorname{dim} H^{0}(M, L)^{G}>0$, then necessarily $\chi_{K, L}=1$. This condition is independent of the choice of $K \in(K)$.

Corollary 3.2. Suppose that $M$ is a complex projective $G$-manifold, and let $(K) \in \operatorname{Conj}(G)$ be the principal orbit type of the action of $G$ on $M$. Let

$$
\mathcal{C} \subseteq \mathrm{C}^{G}(M) \subseteq \mathrm{NS}^{G}(M)_{\mathbb{R}}
$$

be an open chamber in the $G$-ample cone (so that every $L \in \mathrm{Pic}^{G}(M)$ whose numerical class $[L]$ belongs to $\mathcal{C}$ is ample and regular). Then there exists a 
constant $C=C_{\mathcal{C}}>0$ such that the following holds: Suppose $L \in \operatorname{Pic}^{G}(M)$, $[L] \in \mathcal{C}$, and let $(K)$ be the principal orbit type of the $G$-action on $\Phi_{L}^{-1}(0)$. If $\chi_{K, L}=1$, then

$$
\operatorname{dim} H^{0}\left(M, L^{\otimes k}\right)^{G}=C \operatorname{vol}\left(M_{0}(\mathcal{C}), \Omega_{0}(L)\right) k^{\mathrm{n}-\mathrm{g}}+\text { L.O.T. }
$$

as $k \rightarrow+\infty$.

Remark 3.2. Here $M_{0}(\mathcal{C})$ denotes the GIT quotient of $M$ with respect to any of the GIT-equivalent line bundles whose numerical class belongs to the open chamber $\mathcal{C}$. In particular, the Kähler form $\Omega_{0}(L)$ on $M_{0}(\mathcal{C})$ depends linearly on $L$ as $L$ varies among the $G$-linearized line bundles class belongs to $\mathcal{C}$. Furthermore, as we argue below the principal orbit type $(K)$ does not depend on the choice of $[L] \in \mathcal{C}$, so that the condition $\chi_{K, L \otimes k}=1$ is satisfied by any $[L] \in \mathcal{C}$, for some fixed integer $k>0$.

Proof of Corollary [3.2, For $[l] \in \mathcal{C}$, let $\Phi_{[l]}: M \rightarrow \mathfrak{g}^{*}$ denote the associated Hamiltonian structure. Let us set $P_{[l]}=\left(\Phi_{[l]}\right)^{-1}(0)$. Then the submanifolds $P_{[l]}=\Phi_{[l]}^{-1}(0)$ form a smooth family of $G$-manifolds as $[l]$ varies in $\mathcal{C}$. In view of the rigidity of compact group actions GGK], this family is locally equivariantly trivial. Thus, $(K)$ is the principal orbit type of any $\Phi_{[l]}^{-1}(0)$.

For $[l] \in \mathcal{C}$, let us now define $\widetilde{P}_{[l], \mu}$ as in (9) , and let $\widetilde{P}_{[l], \mu,(K)}=\bigcup_{i} \widetilde{P}_{[l], \mu,(K), i}$ be the union of the connected components of $\widetilde{P}_{[l], \mu}$ mapping dominantly onto $M_{0}(L)=M_{0}(\mathcal{C})$. Let us set again $\Sigma_{[l], \mu}=\widetilde{P}_{[l], \mu} / G, \Sigma_{[l], \mu, K}=\widetilde{P}_{[l], \mu,(K)} / G$, $\Sigma_{[l], \mu,(K), i}=: \widetilde{P}_{[l], \mu,(K), i} / G$.

For every $i$, let $\mathrm{d}_{i}$ the cardinality of the generic stabilizer subgroup of a point in $\widetilde{P}_{\mu,(K), i}$, and let $\mathrm{g}_{i}$ be the degree of the unramified covering $\Sigma_{[l], \mu,(K), i} \rightarrow$ $M_{0}(\mathcal{C})$. By the above, the $\Sigma_{[l], \mu}$ form a smooth family as $[l]$ varies in $\mathcal{C}$. It follows that the sum $C=\sum_{i} \mathrm{~g}_{i} / \mathrm{d}_{i}$ only depends on $\mathcal{C}$. The statement follows from this, by arguing as in the proof of Theorem 3.1.

Let us now only assume that $L \in \operatorname{Pic}^{G}(M)$ is ample and non-empty stable locus. Let $f: \widetilde{M} \rightarrow M$ be a Kirwan resolution of $(M, L)$. Thus, $f^{*}(L)^{\otimes k}(-E) \in \operatorname{Pic}^{G}(\widetilde{M})$ is ample and regular for every $k \gg 0$, where $E$ is some $f$-exceptional $G$-invariantly effective divisor. Let $\widetilde{C}$ be the chamber in the $G$-ample cone of $\widetilde{M}$ containing the numerical class of $f^{*}(L)^{\otimes k}(-E)$, $k \gg 0[\mathrm{DH}],\left[\mathrm{Th}\right.$. Let $\widetilde{M}_{0}(\widetilde{C})$ be GIT quotient of $\widetilde{M}$ with respect to $\widetilde{C}$. The line bundle $f^{*}(L) \in \operatorname{Pic}^{G}(\widetilde{M})$ descends to a line orbi-bundle $L_{0}$ on $\widetilde{M}_{0}(\widetilde{C})$; let $\widetilde{\Omega}_{0}$ be a 2 -form representing its first Chern class.

Corollary 3.3. Suppose that $L \in \operatorname{Pic}^{G}(M)$ is ample and has non-empty stable locus. Let $\mu$ be a dominant weight. Assume again for simplicity that 
the stabilizer of a general $p \in M$ is a central subgroup. If $L$ and $\mu$ are not numerically compatible, then $H_{\mu}^{0}\left(M, L^{\otimes k}\right)=0$ for every $k=1,2, \ldots$ If on the other hand $L$ and $\mu$ are numerically compatible, then

$$
\operatorname{vol}_{\mu}(L)=\operatorname{dim}\left(V_{\mu}\right)^{2} \cdot \operatorname{vol}\left(\widetilde{M}_{0}(\widetilde{C}), \widetilde{\Omega}_{0}\right)>0 .
$$

Proof. Suppose $p \gg 0$ is such that $L^{\otimes p e_{G}(L)}(-E)$ is ample and regular; we may then find $r \gg 0$ such that $H^{0}\left(\widetilde{M}, L^{\otimes p r e} G(L)(-r E)\right)^{G} \neq 0$. Let $p_{i} \uparrow+\infty$ be a sequence of integers prime with $e_{G}(L)$. It follows from Proposition 5.1 below that $\operatorname{vol}_{\mu}\left(L^{\otimes p_{i}}\right)=p_{i}^{\mathrm{n}-\mathrm{g}} \operatorname{vol}_{\mu}(L)$. Therefore,

$$
\frac{\operatorname{vol}_{\mu}\left(f^{*}(L)^{\otimes p_{i}}(-E)\right)}{p_{i}^{\mathrm{n}-\mathrm{g}}} \leq \operatorname{vol}_{\mu}(L) \leq \frac{\operatorname{vol}_{\mu}\left(f^{*}(L)^{\otimes p_{i}+p r e_{G}(L)}(-r E)\right)}{p_{i}^{\mathrm{n}-\mathrm{g}}} .
$$

The statement follows from (15) by taking the limit as $i \rightarrow+\infty$.

Let us now briefly consider the more general case where $L \in \operatorname{Pic}^{G}(M)$ is nef and big, and has positive 0-volume. Let us suppose, for simplicity, that there exists $B \in \operatorname{Pic}^{G}(M)$ ample and such that $M^{\mathrm{s}}(B) \neq \emptyset$, $\operatorname{codim}\left(M^{\mathrm{u}}(B)\right) \geq 2$. Then by Corollary 6.6 below there exist a $G$-invariantly effective $E \in \operatorname{Pic}^{G}(M)$ such that $L^{\otimes k}(-E)$ is ample and $M^{\mathrm{s}}\left(L^{\otimes k}(-E)\right) \neq \emptyset$ for every $k \gg 0$. After replacing $E$ by some power, we can fix $k_{0} \gg 0$ divisible by $e_{G}(L)$ and such that $H^{0}\left(M, L^{\otimes k_{0}}(-E)\right)^{G} \neq\{0\}$. Letting $p_{i} \uparrow+\infty$ be a sequence of integers prime with $e_{G}(L)$, we obtain

$$
\frac{\operatorname{vol}_{\mu}\left(L^{\otimes p_{i}}(-E)\right)}{p_{i}^{\mathrm{n}-\mathrm{g}}} \leq \operatorname{vol}_{\mu}(L) \leq \frac{\operatorname{vol}_{\mu}\left(L^{\otimes p_{i}+k_{0}}(-E)\right)}{p_{i}^{\mathrm{n}-\mathrm{g}}} .
$$

If $B$ is regular, it may be assumed that so is $L^{\otimes k}(-E)$, and that it eventually belongs to a fixed chamber in the $G$-ample cone of $M$. Then by passing to the limit as $i \rightarrow+\infty$ we obtain a statement formally similar to the one in Theorem [3.1. Otherwise, we may still apply a Kirwan resolution and reach a statement as in Corollary 3.3, we shall leave it to the reader to complete the argument.

\section{Finiteness}

Unlike the good cases considered in section \$3, in general equivariant volumes and their dependence on the weight $\mu$ will have a less controlled behavior. 
Example 4.1. Suppose $\operatorname{dim}(G)>0$. Let $M$ be a complex projective variety, and $L$ an ample line bundle on $M$. Let $G$ act trivially on $M$ and $L$. Then $\operatorname{vol}_{0}(L)=+\infty$ and $\operatorname{vol}_{\mu}(L)=0$ for all $\mu \neq 0$. Notice that in this case $M^{\mathrm{u}}(L)=M^{\mathrm{s}}(L)=\emptyset$, and $M=M^{\mathrm{ss}}(L)$.

Example 4.2. Consider the linear action $S^{1} \times \mathbb{C}^{\mathrm{n}+1} \rightarrow \mathbb{C}^{\mathrm{n}+1}$ given by

$$
t \cdot\left(z_{0}, \ldots, z_{\mathrm{n}}\right)=\left(z_{0}, t z_{1}, \ldots, t z_{\mathrm{n}}\right) .
$$

This defines an action on $\mathbb{P}^{n}$ with an obvious linearization to the hyperplane bundle. If $\mathrm{n}>1$ then $\operatorname{vol}_{\mu}\left(\mathcal{O}_{\mathbb{P}^{\mathrm{n}}}(1)\right)=0$ for every $\mu$. In this case, $M^{\mathrm{ss}}(L)=$ $\mathbb{P}^{\mathrm{n}} \backslash\left\{z_{0}=0\right\}, M^{\mathrm{s}}(L)=\emptyset$.

Example 4.3. Consider next the linear action $S^{1} \times \mathbb{C}^{\mathrm{n}+1} \rightarrow \mathbb{C}^{\mathrm{n}+1}$ given by

$$
t \cdot\left(z_{0}, \ldots, z_{\mathrm{n}}\right)=\left(z_{0}, z_{1}, \ldots, z_{\mathrm{n}-1}, t z_{\mathrm{n}}\right),
$$

with the induced action on $\mathbb{P}^{\mathrm{n}}$ and the tautological linearization on the hyperplane bundle. Now $M^{\mathrm{u}}\left(\mathcal{O}_{\mathbb{P}^{\mathrm{n}}}(1)\right)=\{[0: \cdots: 0: 1]\}, M^{\mathrm{s}}\left(\mathcal{O}_{\mathbb{P}^{\mathrm{n}}}(1)\right)=\emptyset$. We have $\operatorname{vol}_{\mu}\left(\mathcal{O}_{\mathbb{P}^{n}}(1)\right)=1$ for every $\mu \geq 0, \operatorname{vol}_{\mu}\left(\mathcal{O}_{\mathbb{P}^{n}}(1)\right)=0$ if $\mu<0$.

We shall now give some general hypothesis guaranteeing that every $G$-line bundle on $M$ satisfies $\operatorname{vol}_{\mu}(L)<+\infty$ for every maximal weight $\mu$.

Proposition 4.1. Suppose that there exists a regular ample $G$-line bundle $B$ on $M$. Then $\operatorname{vol}_{\mu}(L)<+\infty$ for every $L \in \operatorname{Pic}^{G}(M)$ and every maximal weight $\mu$.

Proof. By Corollary 3.1, we may find $k=e l \gg 0$ such that $H^{0}\left(M, B^{\otimes k}\right)^{G} \neq$ $\{0\}$. By Remark [2.6, $\operatorname{vol}_{\mu}(L) \leq \operatorname{vol}_{\mu}\left(L \otimes B^{\otimes k}\right)$.

Hence it suffices to show that if $k=e l \gg 0$ then $\operatorname{vol}_{\mu}\left(L \otimes B^{\otimes k}\right)<+\infty$ for every $\mu$.

If $k \gg 0$ the $G$-line bundle $L \otimes B^{\otimes k}$ is ample and its equivalence class lies in the interior of the same chamber as $B$; in particular, it is regular. The statement follows by Theorem 3.1 .

Corollary 4.1. Suppose that there exists an ample G-line bundle $A$ on $M$ such that $M^{\mathrm{S}}(A) \neq \emptyset$. Then $\operatorname{vol}_{\mu}(L)<+\infty$ for every $L \in \operatorname{Pic}^{G}(M)$ and every maximal weight $\mu$.

Proof. Let $f: \tilde{M} \rightarrow M$ be a Kirwan resolution of the pair $(M, A)$ (Definition 2.9). By definition, there exists an ample and regular $G$-line bundle on $\tilde{M}$. Hence $\operatorname{vol}_{\eta}(N)<+\infty$ for every $G$-line bundle $N$ on $\tilde{M}$ and every maximal weight $\eta$. 
Now suppose $L \in \operatorname{Pic}^{G}(M)$. There is a $G$-equivariant isomorphism $f_{*}\left(f^{*}(L)\right)^{\otimes k} \cong L^{\otimes k}$, whence $G$-equivariant isomorphisms

$$
H^{0}\left(\tilde{M}, f^{*}(L)^{\otimes k}\right) \cong H^{0}\left(M, L^{\otimes k}\right)
$$

for every $k=1,2, \ldots$ Therefore, given any maximal weight $\mu$, we have $\operatorname{vol}_{\mu}(L)=\operatorname{vol}_{\mu}\left(f^{*}(L)\right)<+\infty$.

If on the other hand an ample $G$-line bundle $L$ has no semi-stable points, then all equivariant volumes of $L$ vanish.

Proposition 4.2. Let $M$ be a projective G-variety. Let $L \in \operatorname{Pic}^{G}(M)$ be ample and such that $M=M^{\mathrm{u}}(L)$. Then for every maximal weight $\mu$ there exists $r_{\mu} \geq 0$ such that $H^{0}\left(M, L^{\otimes r}\right)_{\mu}=0$ for every integer $r \geq r_{\mu}$. In particular, $\operatorname{vol}_{\mu}(L)=0$ for every maximal weight $\mu$.

Proof of Proposition 4.2. Let $\Phi_{L}: M \rightarrow \mathfrak{g}^{*}$ be a moment map for $L$ (Definition 2.6] and Remark 2.3); then $\Phi_{L^{\otimes r}}=: r \Phi_{L}$ is a moment map for $L^{\otimes r}$. By the hypothesis, $\Phi_{L}$ is bounded in norm away from zero Ki1. Therefore, there exists $c>0$ such that

$$
\min \left\{\left\|\Phi_{L \otimes r}(m)\right\|: m \in M\right\} \geq c r .
$$

Given any $\mu \in \mathfrak{g}^{*}$ we then have $\mu \notin r \Phi_{L}(M)=\Phi_{L \otimes r}(M)$ if $r \gg 0$.

If $M$ is a projective manifold, Theorem 6.3 of [GS3] implies $H^{0}\left(M, L^{\otimes r}\right)_{\mu}=$ $\{0\}$ if $r \gg 0$.

If on the other hand $M$ is singular, let us choose a $G$-equivariant resolution of singularities, $f: \tilde{M} \rightarrow M$. There exists a $G$-invariantly effective exceptional normal crossing divisor $E \subseteq \tilde{M}$ such that $f^{*}(L)^{\otimes r}(-E) \in \operatorname{Pic}^{G}(\tilde{M})$ is ample for every $r \gg 0$. Let us consider the $G$-equivariant short exact sequence

$$
0 \longrightarrow f^{*}(L)^{\otimes r}(-E) \longrightarrow f^{*}(L)^{\otimes r} \longrightarrow \mathcal{O}_{E} \otimes f^{*}(L)^{\otimes r} \longrightarrow 0
$$

At every maximal weight $\mu$, this induces an exact sequence

$0 \longrightarrow H^{0}\left(\tilde{M}, f^{*}(L)^{\otimes r}(-E)\right)_{\mu} \longrightarrow H^{0}\left(\tilde{M}, f^{*}(L)^{\otimes r}\right)_{\mu} \longrightarrow H^{0}\left(E, \mathcal{O}_{E} \otimes f^{*}(L)^{\otimes r}\right)_{\mu}$.

A moment map for $f^{*}(L)^{\otimes r}(-E)$ has the form $\Phi_{r}=r \Phi_{L} \circ f+\Phi_{0}: \tilde{M} \rightarrow \mathfrak{g}^{*}$, where $\Phi_{0}: M \rightarrow \mathfrak{g}^{*}$ is a constant equivariant map. It follows from (16) that $\mu \notin \Phi_{r}(\tilde{M})$ if $r \gg 0$, and therefore $H^{0}\left(\tilde{M}, f^{*}(L)^{\otimes r}(-E)\right)_{\mu}=0$, again by Theorem 6.3 of GS3. 
Next let us decompose $E$ as the sum of its irreducible components: $E=$ $\sum_{j} E_{j}$. Each $E_{j}$ is an irreducible $G$-invariant subscheme of $\tilde{M}$, and there is an equivariant injection $\mathcal{O}_{E} \rightarrow \bigoplus_{j} \mathcal{O}_{E_{j}}$. Therefore, it suffices to prove that $H^{0}\left(E_{j}, \mathcal{O}_{E_{j}} \otimes f^{*}(L)^{\otimes r}\right)_{\mu}=0$ for every $j$ and every $r \gg 0$. If all the $E_{j}$ 's are reduced, hence non-singular varieties, the statement follows from Theorem 2.1. If some of the $E_{j}$ 's are not reduced, the same conclusion is reached by filtering $\mathcal{O}_{E_{j}} \otimes f^{*}(L)^{\otimes r}$ by line bundles on the underlying reduced manifold. More precisely, suppose to fix ideas that $E_{j}$ has generic multiplicity two, and write $E_{j}=2 F_{j}$, where $F_{j}$ is codimension one $G$-invariant irreducible complete submanifold o $M$. We have an equivariant short exact sequence

$$
0 \longrightarrow \mathcal{O}_{F_{j}}\left(L^{\otimes k}\left(-F_{j}\right)\right) \longrightarrow \mathcal{O}_{E_{j}} \otimes L^{\otimes k} \longrightarrow \mathcal{O}_{E_{j}}\left(L^{\otimes k}\right) \longrightarrow 0 .
$$

By Theorem 2.1 and Lemma 2.2.

$$
H^{0}\left(F_{j}, \mathcal{O}_{F_{j}}\left(L^{\otimes k}\left(-F_{j}\right)\right)\right)_{\mu}=0 \text { and } H^{0}\left(F_{j}, \mathcal{O}_{F_{J}}\left(L^{\otimes k}\right)\right)_{\mu}=0
$$

for every $r \gg 0$. The general case is similar.

We shall need the following auxiliary result:

Proposition 4.3. Let $M$ be a complex projective G-manifold. Suppose that $L \in \operatorname{Pic}^{G}(M)$ is ample and has non-empty stable locus: $M^{\mathrm{s}}(L) \neq \emptyset$. Let $e=e_{G}(L)$ be the $G$-exponent of $L$ (Definition 2.14). Suppose $0 \neq \sigma \in$ $H^{0}\left(M, L^{\otimes k e}\right)^{G}$ is general, for some $k \gg 0$. Let $Z=\operatorname{div}(\sigma) \subseteq M$ be the corresponding divisor. Then

$$
\operatorname{vol}_{\mu}\left(Z,\left.A\right|_{Z}\right)<+\infty
$$

for every $A \in \operatorname{Pic}^{G}(M)$ which is GIT-equivalent to $L$ and for every maximal weight $\mu$.

Remark 4.1. Recall that ample $A, L \in \operatorname{Pic}^{G}(M)$ are said to be GIT-equivalent if $M^{\mathrm{u}}(A)=M^{\mathrm{u}}(L), M^{\mathrm{s}}(A)=M^{\mathrm{s}}(L)[\overline{\mathrm{DH}}]$. However, the statement of the Proposition still holds if we only require $M^{\mathrm{u}}(A) \supseteq M^{\mathrm{u}}(L)$.

Proof of Proposition 4.3 . We may decompose the $G$-invariantly effective divisor $Z$ as $Z=Z_{\mathrm{u}}+Z_{\mathrm{s}}$, where $Z_{\mathrm{u}}, Z_{\mathrm{s}}$ are $G$-invariantly effective divisors, $Z_{\mathrm{u}}$ is supported on the unstable locus $M^{\mathrm{u}}(L)$, and no irreducible component of $Z_{\mathrm{s}}$ is supported on $M^{\mathrm{u}}(L)$. Since we may assume that the base locus of $H^{0}\left(M, L^{\otimes k e}\right)^{G}$ is $M^{\mathrm{u}}(L)$, we may also suppose without loss that every irreducible component of $Z_{\mathrm{s}}$ intersects the stable locus $M^{\mathrm{s}}(L)$. 
Since $Z, Z_{\mathrm{u}}$ and $Z_{\mathrm{s}}$ are all $G$-invariant subschemes of $M$, there is a $G$ equivariant injection $\mathcal{O}_{Z} \hookrightarrow \mathcal{O}_{Z_{\mathrm{u}}} \oplus \mathcal{O}_{Z_{\mathrm{s}}}$. Tensoring by $A^{\otimes k}$, and passing to global sections, for every maximal weight $\mu$ this implies

$$
h_{\mu}^{0}\left(Z, A^{\otimes k} \otimes \mathcal{O}_{Z}\right) \leq h_{\mu}^{0}\left(Z_{\mathrm{u}}, A^{\otimes k} \otimes \mathcal{O}_{Z_{\mathrm{u}}}\right)+h_{\mu}^{0}\left(Z_{\mathrm{s}}, A^{\otimes k} \otimes \mathcal{O}_{Z_{\mathrm{s}}}\right)
$$

$\left(\right.$ where $\left.h_{\mu}^{0}=: \operatorname{dim} H_{\mu}^{0}\right)$. Thus,

$$
\operatorname{vol}_{\mu}\left(Z,\left.A\right|_{Z}\right) \leq \operatorname{vol}_{\mu}\left(Z_{\mathrm{u}},\left.A\right|_{Z_{\mathrm{u}}}\right)+\operatorname{vol}_{\mu}\left(Z_{\mathrm{s}},\left.A\right|_{Z_{\mathrm{s}}}\right) .
$$

Let us decompose $Z_{\mathrm{s}}$ as the sum of its irreducible components, $Z_{\mathrm{s}}=$ $\sum_{j} Z_{\mathrm{s} j}$. By the generality of $\sigma$ and Bertini's Theorem, every $Z_{\mathrm{s} j}$ is reduced and non-singular away from $M^{\mathrm{u}}(L)$. Arguing as above, we have

$$
h_{\mu}^{0}\left(Z_{\mathrm{s}}, A^{\otimes k} \otimes \mathcal{O}_{Z_{\mathrm{s}}}\right) \leq \sum_{j} h_{\mu}^{0}\left(Z_{\mathrm{s} j}, A^{\otimes k} \otimes \mathcal{O}_{Z_{\mathrm{s} j}}\right),
$$

whence $\operatorname{vol}_{\mu}\left(Z_{\mathrm{s}},\left.A\right|_{Z_{\mathrm{s}}}\right) \leq \sum_{j} \operatorname{vol}_{\mu}\left(Z_{\mathrm{s} j},\left.A\right|_{Z_{\mathrm{s} j}}\right)$.

Lemma 4.1. $\operatorname{vol}_{\mu}\left(Z_{\mathrm{s} j},\left.A\right|_{Z_{\mathrm{s} j}}\right)<+\infty$ for every $j$ and $\mu$.

Proof of Lemma 4.1. Let us assume, to begin with, that the $Z_{\mathrm{s} j}$ 's are nonsingular. Since $M^{\mathrm{s}}\left(\left.L\right|_{Z_{\mathrm{s} j}}\right)=Z_{\mathrm{s} j} \cap M^{\mathrm{s}}(L) \neq \emptyset$ by construction, the statement follows from Corollary 4.1 .

In general, let us choose for every $j$ a $G$-equivariant resolution of singularities, $f_{j}: \tilde{Z}_{\mathrm{s} j} \rightarrow Z_{\mathrm{s} j}$, which may be assumed to be an isomorphism away from $Z_{\mathrm{s} j} \cap M^{\mathrm{u}}(L)[\mathrm{EV}],[\mathrm{EH}]$. We may find a $G$-invariant effective exceptional divisor $E_{j} \subseteq \tilde{Z}_{\mathrm{s} j}$ such that $B_{j}=: f_{j}^{*}(L)^{\otimes k}\left(-E_{j}\right)$ is an ample $G$-divisor on $\tilde{Z}_{\mathrm{s} j}$ for every $k \gg 0$. Clearly, $f_{j}\left(E_{j}\right) \subseteq Z_{\mathrm{s} j} \cap M^{\mathrm{u}}(L)$. We have:

Claim 4.1. $M^{\mathrm{s}}\left(B_{j}\right) \neq \emptyset$ for all $k \gg 0$.

Proof of Claim 4.1. Since $L$ is ample, section restriction $H^{0}\left(M, L^{\otimes k}\right) \rightarrow$ $H^{0}\left(Z_{\mathrm{s} j}, L^{\otimes k} \otimes \mathcal{O}_{Z_{\mathrm{s} j}}\right)$ is a $G$-equivariant surjective linear map, for every $k \gg 0$. On the other hand, for $k$ large and divisible, by definition we have:

$$
\operatorname{Bs}\left(\left|H^{0}\left(M, L^{\otimes k}\right)^{G}\right|\right)=M^{\mathrm{u}}(L) .
$$

Since furthermore by Nagata's Theorem the algebra

$$
R(L)^{G}=: \bigoplus_{k \geq 0} H^{0}\left(M, L^{\otimes k}\right)^{G}
$$

is finitely generated, the order of vanishing of the general $\tau \in H^{0}\left(M, L^{\otimes k}\right)^{G}$ along $M^{\mathrm{u}}(L)$ grows to infinity as $k \rightarrow+\infty$; by the above, the same then holds of the order of vanishing of the general section in $H^{0}\left(Z_{\mathrm{s} j}, L^{\otimes k} \otimes \mathcal{O}_{Z_{\mathrm{s} j}}\right)^{G}$. On the upshot, we have: 
i): every invariant section of $f_{j}^{*}(L)^{\otimes k}$ coming from the linear map

$$
H^{0}\left(M, L^{\otimes k}\right)^{G} \rightarrow H^{0}\left(Z_{\mathrm{s} j}, L^{\otimes k} \otimes \mathcal{O}_{Z_{\mathrm{s} j}}\right)^{G} \hookrightarrow H^{0}\left(\tilde{Z}_{\mathrm{s} j}, f_{j}^{*}(L)^{\otimes k}\right)^{G}
$$

actually lives in the image of the injection

$$
H^{0}\left(\tilde{Z}_{\mathrm{s} j}, f_{j}^{*}(L)^{\otimes k}\left(-E_{j}\right)\right)^{G} \hookrightarrow H^{0}\left(\tilde{Z}_{\mathrm{s} j}, f_{j}^{*}(L)^{\otimes k}\right)^{G} ;
$$

ii): when viewed as an invariant global section of $B_{j}=f^{*}(L)^{\otimes k}\left(-E_{j}\right)$, every section coming from (19) vanishes along $E_{j}$.

Suppose now $x \in f_{j}^{-1}\left(M^{\mathrm{s}}(L)\right) \subseteq \tilde{Z}_{\mathrm{s} j}$; in particular, $x \notin E_{j}$. Since the stabilizer of $f(x)$ is discrete, so is the stabilizer of $x$. For $k$ large and divisible, furthermore, there exists $s \in H^{0}\left(M, L^{\otimes k}\right)^{G}$ such that $s(x) \neq 0$ and the $\tilde{G}_{-}$ orbit is closed in $M_{s}=\{p \in M: s(p) \neq 0\}$. When we pull-back $s$ to a section of $f_{j}^{*}(L)^{\otimes k}\left(-E_{j}\right)$ using i), the same then holds in view of ii) and the fact that $f_{j}$ is an isomorphism away from $M^{\mathrm{u}}(L) \cap Z_{\mathrm{s} j}$.

This completes the proof of Claim 4.1.

Lemma 4.1 now follows in view of Corollary 4.1. since

$$
\operatorname{vol}_{\mu}\left(Z_{\mathrm{s} j}, A \otimes \mathcal{O}_{Z_{\mathrm{s} j}}\right) \leq \operatorname{vol}_{\mu}\left(\tilde{Z}_{\mathrm{s} j}, f_{j}^{*}(A)\right) .
$$

We conclude that $\operatorname{vol}_{\mu}\left(Z_{\mathrm{s}}, A \otimes \mathcal{O}_{Z_{\mathrm{s}}}\right) \leq \sum_{j} \operatorname{vol}_{\mu}\left(Z_{\mathrm{s} j}, A \otimes \mathcal{O}_{Z_{\mathrm{s} j}}\right)<+\infty$.

The following is where the hypothesis that $A$ and $L$ be GIT-equivalent is being used:

Lemma 4.2. $\operatorname{vol}_{\mu}\left(Z_{\mathrm{u}}, A \otimes \mathcal{O}_{Z_{\mathrm{u}}}\right)=0$ for every maximal weight $\mu$.

Proof of Lemma 4.2. It suffices to prove that $\operatorname{vol}_{\mu}\left(Z_{\mathrm{u} j}, A \otimes \mathcal{O}_{Z_{\mathrm{u} j}}\right)=0$ for every $j$. This follows immediately from Proposition 4.2 when $Z_{\mathrm{u} j}$ is reduced. In the general case, we filter $A \otimes \mathcal{O}_{Z_{\mathrm{u} j}}$ by line bundles on $\left(Z_{\mathrm{u} j}\right)_{\text {red }}$, and argue as in the final part of the proof of Proposition 4.2.

Since $\operatorname{vol}_{\mu}\left(Z, A \otimes \mathcal{O}_{Z}\right) \leq \operatorname{vol}_{\mu}\left(Z_{\mathrm{s}}, A \otimes \mathcal{O}_{Z_{\mathrm{s}}}\right)+\operatorname{vol}_{\mu}\left(Z_{\mathrm{u}}, A \otimes \mathcal{O}_{Z_{\mathrm{u}}}\right)$, the proof of Proposition 4.3 is now complete.

The same argument also proves the following:

Proposition 4.4. Let $M$ be a complex projective $G$-manifold. Suppose that $L \in \operatorname{Pic}^{G}(M)$ is ample and such that $M^{\mathrm{s}}(L) \neq \emptyset$ and $\operatorname{codim}\left(M^{\mathrm{u}}(L)\right) \geq 2$. Let $e=e_{L}$ be the period of $\chi_{K, L}$, as in Corollary [3.1. Suppose $0 \neq \sigma \in$ $H^{0}\left(M, L^{\otimes k e}\right)^{G}$ is general, for some $k \gg 0$. Let $Z=\operatorname{div}(\sigma) \subseteq M$ be the corresponding divisor. Then

$$
\operatorname{vol}_{\mu}\left(Z,\left.R\right|_{Z}\right)<+\infty
$$

for every $R \in \operatorname{Pic}^{G}(M)$ and every maximal weight $\mu$. 


\section{$5 \quad$ Homogeneity Properties}

In this section we shall study the relation between the equivariant volumes of $L$ and $L^{\otimes p}$, where $L \in \operatorname{Pic}^{G}(M)$ and $p \in \mathbb{N}$. Homogeneity does not hold in the most simple-minded sense, as shown by Example 3.1 .

The main result here is Proposition 5.1. First however we need to establish some technical preliminaries.

In the following analysis, we may assume without loss of generality that $M^{\text {ss }}(L) \neq \emptyset$, so that $\mathbb{N}_{G}(L) \neq\{0\}$, for otherwise $\operatorname{vol}_{\mu}(L)=0$ for every $\mu$ in view of Proposition 4.2. This assumption will be implicit throughout.

Definition 5.1. Suppose that $M$ is a complex projective $G$-variety and that $L \in \operatorname{Pic}^{G}(M)$. Let $\mu$ be a maximal weight of $G$. For every integer $f$ with $0 \leq f<e_{G}(L)$ let us define

$$
v_{\mu}(L, f)=: \limsup _{m \rightarrow+\infty} \frac{h_{\mu}^{0}\left(M, L^{\otimes\left(f+m e_{G}(L)\right)}\right)}{\left(f+m e_{G}(L)\right)^{\mathrm{n}-\mathrm{g}} /(\mathrm{n}-\mathrm{g}) !} .
$$

Remark 5.1. $v_{\mu}(L, f)$ may be defined in the same manner for any $f \in \mathbb{Z}$, and with this interpretation it only depends on the class of $f \bmod e_{G}(L)$. At places we shall refer to this alternative interpretation, but this should cause no confusion.

It is clear from the definition that $v_{\mu}(L, f) \leq \operatorname{vol}_{\mu}(L)$ for every $f \in$ $\left\{0,1, \ldots, e_{G}(L)-1\right\}$. On the other hand, suppose that $k_{i} \uparrow+\infty$ is a sequence of integers such that

$$
\lim _{i \rightarrow+\infty} \frac{(\mathrm{n}-\mathrm{g}) !}{k_{i}^{\mathrm{n}-\mathrm{g}}} h_{\mu}^{0}\left(M, L^{\otimes k_{i}}\right)=\operatorname{vol}_{\mu}(L) .
$$

Perhaps after passing to a subsequence, we may assume without loss of generality that the $k_{i}$ 's are constant $\bmod e_{G}(L)$. Thus, if $f_{0} \in\left\{0,1, \ldots, e_{G}(L)-1\right\}$ and $k_{i} \equiv f_{0}\left(\bmod . e_{G}(L)\right)$ for every $i$, then clearly $v_{\mu}\left(L, f_{0}\right)=\operatorname{vol}_{\mu}(L)$. Thus,

Lemma 5.1. In the hypothesis of Definition 5.1,

$$
\operatorname{vol}_{\mu}(L)=\max \left\{v_{\mu}(L, f): 0 \leq f<e_{G}(L)\right\} .
$$

The key to establishing the homogeneity properties of $\operatorname{vol}_{\mu}$ is the following:

Lemma 5.2. In the hypothesis of Definition 5.1, for every $f \in\left\{0,1, \ldots, e_{G}(L)-\right.$ 1) and any $p \in \mathbb{N}$, we have

$$
v_{\mu}(L, f)=(\mathrm{n}-\mathrm{g}) ! \limsup _{k \rightarrow+\infty} \frac{h_{\mu}^{0}\left(M, L^{\otimes\left(f+k p e_{G}(L)\right)}\right)}{\left(f+k p e_{G}(L)\right)^{\mathrm{n}-\mathrm{g}}} .
$$


Proof of Lemma 5.2. For any integer $r \geq 0$, let us define

$$
u_{r}=:(\mathrm{n}-\mathrm{g}) ! \limsup _{k \rightarrow+\infty} \frac{h_{\mu}^{0}\left(M, L^{\otimes}\left(f+(k p+r) e_{G}(L)\right)\right.}{\left(f+(k p+r) e_{G}(L)\right)^{\mathrm{n}-\mathrm{g}}} .
$$

Clearly, $u_{r}=u_{r+p}$ for every $r$, and for every $r_{0} \in \mathbb{N}$ one has $v_{\mu}(L, f)=$ $\max \left\{u_{r_{0}+1}, \ldots, u_{r_{0}+p}\right\}$. We are reduced to proving the following:

Claim 5.1. There exists $r_{0} \in \mathrm{N}$ such that $u_{r}=u_{0}$ for all $r \in\left\{r_{0}+1, \ldots, r_{0}+\right.$ $p\}$.

Proof of Claim 5.1. Let us choose $r_{0} \in \mathbb{N}$ such that for every $r \geq r_{0}$ we have $H^{0}\left(M, L^{\otimes r e_{G}(L)}\right)^{G} \neq\{0\}$, for every $r \geq r_{0}$. We shall argue that the statement holds for $r_{0}$. To see this, let us choose $q \in \mathbb{N}$ such that $q p-\left(r_{0}+\right.$ $p)>r_{0}$. For every $r \in\left\{r_{0}+1, \ldots, r_{0}+p\right\}$, we have $H^{0}\left(M, L^{\otimes r e_{G}(L)}\right)^{G} \neq\{0\}$ and $H^{0}\left(M, L^{\otimes(q p-r) e_{G}(L)}\right)^{G} \neq\{0\}$. By the usual argument, this implies

$h_{\mu}^{0}\left(M, L^{\otimes}\left(f+k p e_{G}(L)\right)\right) \leq h_{\mu}^{0}\left(M, L^{\otimes\left(f+(k p+r) e_{G}(L)\right)}\right) \leq h_{\mu}^{0}\left(M, L^{\otimes\left(f+(k+q) p e_{G}(L)\right)}\right)$.

Finally, dividing by $\left(f+k p e_{G}(L)\right)^{\mathrm{n}-\mathrm{g}} /(\mathrm{n}-\mathrm{g})$ ! and passing to limsup for $k \rightarrow+\infty$, we are done.

Corollary 5.1. In the hypothesis of Definition [5.1, for every $f \in\left\{0,1, \ldots, e_{G}(L)-\right.$ 1) and any $p \in \mathbb{N}$, we have

$$
v_{\mu}\left(L^{\otimes p}, f\right)=p^{\mathrm{n}-\mathrm{g}} v_{\mu}(L, p f) .
$$

Proof. By definition,

$$
\begin{aligned}
v_{\mu}\left(L^{\otimes p}, f\right) & =(\mathrm{n}-\mathrm{g}) ! \limsup _{m \rightarrow+\infty} \frac{h_{\mu}^{0}\left(M, L^{\otimes p\left(f+m e_{G}\left(L^{\otimes p}\right)\right)}\right)}{\left(f+m e_{G}\left(L^{\otimes p}\right)\right)^{\mathrm{n}-\mathrm{g}}} \\
& =(\mathrm{n}-\mathrm{g}) ! p^{\mathrm{n}-\mathrm{g}} \limsup _{m \rightarrow+\infty} \frac{h_{\mu}^{0}\left(M, L^{\otimes\left(p f+m p e_{G}\left(L^{\otimes p}\right)\right)}\right)}{\left(p f+m p e_{G}\left(L^{\otimes p}\right)\right)^{\mathrm{n}-\mathrm{g}}} .
\end{aligned}
$$

By Lemma 2.3. we have $e_{G}\left(L^{\otimes p}\right)=e_{G}(L) / \operatorname{gcd}\left(e_{G}(L), p\right)$. Let us set $q=: p / \operatorname{gcd}\left(e_{G}(L), p\right)$, so that $p e_{G}\left(L^{\otimes p}\right)=q e_{G}(L)$. Inserting this in (20) and applying Lemma 5.2 we obtain

$$
\begin{aligned}
v_{\mu}\left(L^{\otimes p}, f\right) & =p^{\mathrm{n}-\mathrm{g}}(\mathrm{n}-\mathrm{g}) ! \limsup _{m \rightarrow+\infty} \frac{h_{\mu}^{0}\left(M, L^{\otimes\left(p f+m q e_{G}(L)\right)}\right)}{\left(p f+m q e_{G}(L)\right)^{\mathrm{n}-\mathrm{g}}} \\
& =p^{\mathrm{n}-\mathrm{g}} v_{\mu}(L, p f) .
\end{aligned}
$$

Now we can prove 
Proposition 5.1. Suppose that $L \in \operatorname{Pic}^{G}(M)$ and that $p \in \mathbb{N}$ is prime with $e_{G}(L)$. Then $\operatorname{vol}_{\mu}\left(L^{\otimes p}\right)=p^{\mathrm{n}-\mathrm{g}} \operatorname{vol}_{\mu}(L)$.

Proof of Proposition 5.1. It is immediate from the definition of equivariant volume that $\operatorname{vol}_{\mu}\left(L^{\otimes p}\right) \leq p^{\mathrm{n}-\mathrm{g}} \operatorname{vol}_{\mu}(L)$.

To verify the reverse inequality, let us choose $f \in \mathbb{N}$ such that $\operatorname{vol}_{\mu}(L)=$ $v_{\mu}(L, f)$ (Lemma 5.1). Since $v_{\mu}(L, f)$ only depends on the congruence class of $f$ modulo $e_{G}(L)$, and $p$ is prime with $e_{G}(L)$ by assumption, we may assume $f=p \ell$ for some $\ell \in \mathbb{N}$. Applying Corollary 5.1 we conclude:

$$
\operatorname{vol}_{\mu}(L)=v_{\mu}(L, p \ell)=\frac{v_{\mu}\left(L^{\otimes p}, \ell\right)}{p^{\mathrm{n}-\mathrm{g}}} \leq \frac{\operatorname{vol}_{\mu}\left(L^{\otimes p}\right)}{p^{\mathrm{n}-\mathrm{g}}} .
$$

Corollary 5.2. For any $q \in \mathbb{N}$ we have

$$
\operatorname{vol}_{\mu}\left(L^{\otimes q}\right)=\left(\frac{q}{\operatorname{gcd}\left(q, e_{G}(L)\right)}\right)^{\mathrm{n}-\mathrm{g}} \operatorname{vol}_{\mu}\left(L^{\otimes \operatorname{gcd}\left(q, e_{G}(L)\right)}\right) .
$$

Proof. By Lemma 2.3, we have

$$
e_{G}\left(L^{\otimes \operatorname{gcd}\left(q, e_{G}(L)\right)}\right)=\frac{e_{G}(L)}{\operatorname{gcd}\left(e_{G}(L), \operatorname{gcd}\left(q, e_{G}(L)\right)\right)}=\frac{e_{G}(L)}{\operatorname{gcd}\left(q, e_{G}(L)\right)},
$$

which is relatively prime with $q / \operatorname{gcd}\left(q, e_{G}(L)\right)$. Therefore, the statement follows from Proposition 5.1 given that

$$
\operatorname{vol}_{\mu}\left(L^{\otimes q}\right)=\operatorname{vol}_{\mu}\left(\left(L^{\otimes \operatorname{gcd}\left(q, e_{G}(L)\right)}\right)^{\otimes \frac{q}{\operatorname{gcd}\left(q, e_{G}(L)\right)}}\right) .
$$

Corollary 5.3. For any $p \in \mathbb{N}$, we have

$$
\operatorname{vol}_{\mu}\left(L^{\otimes p e_{G}(L)}\right)=p^{\mathrm{n}-\mathrm{g}} \operatorname{vol}_{\mu}\left(L^{\otimes e_{G}(L)}\right) .
$$

It is in order to single out the case of the trivial representation, which is always homogeneous:

Corollary 5.4. For any $L \in \operatorname{Pic}^{G}(M)$ and any $q \in \mathbb{N}$ we have:

$$
\operatorname{vol}_{0}\left(L^{\otimes q}\right)=q^{\mathrm{n}-\mathrm{g}} \operatorname{vol}_{0}(L) .
$$

Proof. If $p \in \mathbb{N}$ divides $e_{G}(L)$, then

$$
\begin{aligned}
\operatorname{vol}_{0}\left(L^{\otimes p}\right) & =(\mathrm{n}-\mathrm{g}) ! \limsup _{m \rightarrow+\infty} \frac{1}{m^{\mathrm{n}-\mathrm{g}}} h^{0}\left(M, L^{\otimes m p}\right)^{G} \\
& =p^{\mathrm{n}-\mathrm{g}}(\mathrm{n}-\mathrm{g}) ! \limsup _{m \rightarrow+\infty} \frac{1}{(m p)^{\mathrm{n}-\mathrm{g}}} h^{0}\left(M, L^{\otimes m p}\right)^{G} \\
& =p^{\mathrm{n}-\mathrm{g}}(\mathrm{n}-\mathrm{g}) ! \limsup _{m \rightarrow+\infty} \frac{1}{\left(m e_{G}(L)\right)^{\mathrm{n}-\mathrm{g}}} h^{0}\left(M, L^{\otimes m e_{G}(L)}\right)^{G} \\
& =p^{\mathrm{n}-\mathrm{g}} \operatorname{vol}_{0}(L) .
\end{aligned}
$$


Here the third equality holds because $h^{0}\left(M, L^{\otimes m p}\right)^{G}=0$ when $e_{G}(L) \nmid p m$, and the last one holds by definition of $e_{G}(L)$. For any $q \in \mathbb{N}$, we then have by Corollary 5.2 .

$$
\begin{aligned}
\operatorname{vol}_{0}\left(L^{\otimes q}\right) & =\left(\frac{q}{\operatorname{gcd}\left(q, e_{G}(L)\right)}\right)^{\mathrm{n}-\mathrm{g}} \operatorname{vol}_{0}\left(L^{\otimes \operatorname{gcd}\left(q, e_{G}(L)\right)}\right) \\
& =\left(\frac{q}{\operatorname{gcd}\left(q, e_{G}(L)\right)}\right)^{\mathrm{n}-\mathrm{g}} \operatorname{gcd}\left(q, e_{G}(L)\right)^{\mathrm{n}-\mathrm{g}} \operatorname{vol}_{0}(L) \\
& =q^{\mathrm{n}-\mathrm{g}} \operatorname{vol}_{0}(L),
\end{aligned}
$$

where the second equality holds because $\operatorname{gcd}\left(q, e_{G}(L)\right)$ divides $e_{G}(L)$.

\section{Equivariant Kodaira Lemma}

Proposition 6.1. Let $M$ be a complex projective $G$-manifold, and suppose that there exists an ample $B \in \mathrm{Pic}^{G}(M)$ with non-empty stable locus, $M^{\mathrm{s}}(B) \neq \emptyset$, and such that $\operatorname{codim}\left(M^{\mathrm{u}}(B)\right) \geq 2$. Then for every maximal weight $\mu$ and every $L, F \in \operatorname{Pic}^{G}(M)$ such that $\operatorname{vol}_{\mu}(L)>0$ and $H^{0}(M, F)^{G} \neq$ $\{0\}$, one has

$$
\limsup _{k \rightarrow+\infty, k \in \mathbb{N}_{\mu}(L)}\left(\frac{1}{k^{\mathrm{n}-\mathrm{g}}} \operatorname{dim} H^{0}\left(M, L^{\otimes k} \otimes F^{-1}\right)_{\mu}\right)>0 .
$$

In particular, $H^{0}\left(M, L^{\otimes k} \otimes F^{-1}\right)_{\mu} \neq\{0\}$ for arbitrarily large $k \in \mathbb{N}_{\mu}(L)$.

Proof of Proposition 6.1. To begin with, perhaps after passing to a Kirwan resolution of the pair $(M, B)$, we may as well assume that $B$ is ample and regular. Given this, let us first prove the following:

Lemma 6.1. Let $e=e_{G}(B)$ be the $G$-exponent of $B$. Then

$$
H^{0}\left(M, B^{\otimes r e_{G}(B)} \otimes F^{-1}\right)^{G} \neq\{0\}
$$

if $r \gg 0$.

Proof of Lemma 6.1. Let $(K)$ denote the principal orbit type of the $G$-action on $M$, and let $\chi_{K, F}: K \rightarrow S^{1}$ be the character associated to $F \in \operatorname{Pic}^{G}(M)$, as in Remarks 2.13 and 3.1. The hypothesis $H^{0}(M, F)^{G} \neq\{0\}$ implies $\chi_{K, F}=1$. Therefore, the character associated to $B^{\otimes r e_{G}(B)} \otimes F^{-1} \in$ $\operatorname{Pic}^{G}(M)$ is $\left(\chi_{K, B}\right)^{r e_{G}(B)} \cdot \chi_{K, F}^{-1}=\left(\chi_{K, B}\right)^{r e_{G}(B)}=1$.

On the other hand, for $r \gg 0, B^{\otimes r e_{G}(B)} \otimes F^{-1} \in \mathrm{Pic}^{G}(M)$ is ample and regular, as its equivalence class in $\mathrm{NS}^{G}(M)_{\mathbb{R}}$ lies in the interior of the same 
chamber as the class of $B$. Therefore, arguing as in the proof of Theorem 3.1. the dimension of $H^{0}\left(M, B^{\otimes r e_{G}(B)} \otimes F^{-1}\right)^{G}$ may be computed using the Riemann-Roch formula for multiplicities due to Meinrenken.

Now the moment map associated to $B^{\otimes r e} \otimes F^{-1}$ is

$$
\Phi_{B^{\otimes r e} \otimes F^{-1}}=: \operatorname{re} \Phi_{B}-\Phi_{F}: M \rightarrow \mathfrak{g}^{*}
$$

where $\Phi_{F}$ is a moment map for $F$. Consequently, $\Phi_{B^{\otimes r e} \otimes F^{-1}}^{-1}(0) \rightarrow \Phi_{B}^{-1}(0)$, in the following sense: For $\epsilon \in \mathbb{R}$ sufficiently small, let us set $\Phi_{\epsilon}=: \Phi_{B}-\epsilon \Phi_{F}$. Since $0 \in \mathfrak{g}^{*}$ is a regular value of $\Phi_{0}=\Phi_{B}$, and $\Phi_{0}^{-1}(0) \neq \emptyset$, the same holds of $\Phi_{\epsilon}$ for sufficiently small $\epsilon$. Thus, the loci $\Phi_{\epsilon}^{-1}(0) \subseteq M$ form a family of compact connected g-codimensional submanifolds of $M$. The statement of the Lemma now follows from the same asymptotic computations as in the proof of Theorem 3.1 and Corollary 3.2

Given the Lemma, let us choose $0 \neq \tau \in H^{0}\left(M, B^{\otimes r e} \otimes F^{-1}\right)^{G}$ for some $r \gg 0$. Tensor product by $\tau$ determines injective linear maps

$$
H^{0}\left(M, L^{\otimes k} \otimes B^{-r e}\right)_{\mu} \hookrightarrow H^{0}\left(M, L^{\otimes k} \otimes F^{-1}\right)_{\mu}
$$

for every $k$ and $\mu$. We are thus reduced to proving the following:

Claim 6.1. For every maximal weight $\mu$ and every integer $r \gg 0$, there exists a sequence $k_{i} \in \mathbb{N}_{\mu}(L), k_{i} \uparrow+\infty$ such that

$$
\operatorname{dim} H^{0}\left(M, L^{\otimes k_{i}} \otimes B^{-r e}\right)_{\mu}=O\left(k_{i}^{\mathrm{n}-\mathrm{g}}\right) .
$$

Proof of Claim 6.1. Since $+\infty>\operatorname{vol}_{\mu}(L)>0$, we can find a sequence $k_{i} \uparrow+\infty, k_{i} \in \mathbb{N}_{\mu}(L)$, such that

$$
\operatorname{dim} H^{0}\left(M, L^{\otimes k_{i}}\right)_{\mu}=O\left(k_{i}^{\mathrm{n}-\mathrm{g}}\right) .
$$

For $r \gg 0$ and divisible, let us choose a general $\sigma \in H^{0}\left(M, B^{r e}\right)^{G}$, and let $Z_{\sigma}=\operatorname{div}(\sigma)$ be its zero divisor. For every $i$ we have a $G$-equivariant short exact sequence

$$
0 \longrightarrow L^{\otimes k_{i}} \otimes B^{-r e} \longrightarrow L^{\otimes k_{i}} \longrightarrow \mathcal{O}_{Z_{\sigma}}\left(L^{\otimes k_{i}}\right) \longrightarrow 0 .
$$

Passing to the $\mu$-th equivariant summand in cohomology, we obtain the other

$$
0 \rightarrow H^{0}\left(M, L^{\otimes k_{i}} \otimes B^{-r e}\right)_{\mu} \rightarrow H^{0}\left(M, L^{\otimes k_{i}}\right)_{\mu} \rightarrow H^{0}\left(Z_{\sigma}, \mathcal{O}_{Z_{\sigma}}\left(L^{\otimes k_{i}}\right)\right)_{\mu} .
$$

In view of Proposition 4.4, we have $\operatorname{dim} H^{0}\left(Z_{\sigma}, \mathcal{O}_{Z_{\sigma}}\left(L^{\otimes k_{i}}\right)\right)_{\mu}=o\left(k_{i}^{\mathrm{n}-\mathrm{g}}\right)$. The statement of Claim 6.1 follows from (24) in view of (23). 
Corollary 6.1. Let $M$ be a complex projective $G$-manifold, and suppose that there exists an ample $B \in \operatorname{Pic}^{G}(M)$ such that $M^{s}(B) \neq \emptyset, \operatorname{codim}\left(M^{\mathrm{u}}(B)\right) \geq$ 2. Then the following conditions on $L \in \mathrm{Pic}^{G}(M)$ are equivalent:

i): $\operatorname{vol}_{\mu}(L)>0$;

ii): for every $F \in \operatorname{Pic}^{G}(M)$ with $\operatorname{vol}_{0}(F)>0$, there exists a sequence $k_{i} \in$ $\mathbb{N}_{\mu}(L), k_{i} \uparrow+\infty$, such that

$$
L^{\otimes k_{i}}=F^{\otimes e_{G}(F)} \otimes A_{i}
$$

where $\operatorname{dim} H^{0}\left(M, A_{i}\right)_{\mu}=O\left(k_{i}^{\mathrm{n}-\mathrm{g}}\right)$.

Proof of Corollary 6.1. That i) implies ii) is the content of Proposition 6.1. The reverse implication is obvious.

Corollary 6.2. In the hypothesis of Corollary [6.1, if $L \in \mathrm{Pic}^{G}(M)$ satisfies $\operatorname{vol}_{0}(L)>0$, then the underlying line bundle $f_{G}(L) \in \operatorname{Pic}(M)$ is big.

Example 6.1. The following example shows that in general the hypothesis of Corollary 6.1 may not be replaced by the weaker one $M^{\mathrm{s}}(B) \neq \emptyset$ : Let $S^{1}$ act on $\mathbb{C}^{2}$ by $t \cdot\left(z_{0}, z_{1}\right)=:\left(t z_{0}, t^{-1} z_{1}\right)$, and consider the induced action on $\mathbb{P}^{1}$, with the built-in linearization to the hyperplane bundle, $H \in \operatorname{Pic}^{S^{1}}\left(\mathbb{P}^{1}\right)$. Let us consider the product action of $S^{1} \times S^{1}$ on $\mathbb{P}^{1} \times \mathbb{P}^{1}$. Let $\pi_{i}: \mathbb{P}^{1} \times \mathbb{P}^{1} \rightarrow \mathbb{P}^{1}$ be the projections, and define $H_{i}=\pi_{i}^{*}(H) \in \operatorname{Pic}^{G}\left(\mathbb{P}^{1} \times \mathbb{P}^{1}\right)$. Then $\operatorname{vol}_{0}\left(H_{i}\right)=$ $\operatorname{vol}_{0}(H)>0$, but the underlying line bundle of $H_{i}$ is the pull-back by $\pi_{i}$ of the hyperplane bundle on $\mathbb{P}^{1}$, which is not big.

Corollary 6.3. In the situation of Corollary 6.1 suppose $L \in \operatorname{Pic}^{G}(M)$ satisfies $\operatorname{vol}_{0}(L)>0$. Let $(K)$ be the principal orbit type of the action. Then $e_{G}(L)$ equals the period of $\chi_{K, L},\left|\chi_{K, L}\right|$.

Proof of Corollary 6.3. The following argument is inspired by the proof of Corollary 2.2.10 of [L].

If $H^{0}\left(M, L^{\otimes k}\right)^{G} \neq\{0\}$, then $\chi_{K, L^{\otimes k}}=\left(\chi_{K, L}\right)^{k}=1$. Thus, every sufficiently large multiple of $e_{G}(L)$ is a multiple of $\left|\chi_{K, L}\right|$ so that $\left|\chi_{K, L}\right|$ divides $e_{G}(L)$.

To prove that, conversely, $e_{G}(L)$ divides $\left|\chi_{K, L}\right|$, let us replace $L$ by $L^{\otimes \mid} \chi_{K, L} \mid$, so that $\left|\chi_{K, L}\right|=1$; by Lemma 2.3 it suffices to prove that $e_{G}(L)=1$.

Perhaps after passing to a Kirwan resolution of the pair $(M, B)$, we may assume without loss that $B$ is ample and regular. By Lemma 6.1 (or its proof), perhaps after replacing $B$ by $B^{\otimes r_{G}(B)}$ we may assume that $D=$ : $B \otimes L^{-1}$ is $G$-invariantly effective. Since on the other hand $\operatorname{vol}_{0}(L)>0$, by 
Corollary 6.1 we may find $m \gg 0$ such that $L^{\otimes m}$ and $E=: L^{\otimes m} \otimes B^{-1}$ are both $G$-invariantly effective (recall the choice of the exponents $k_{i}$ described in (23) $)$. Thus,

$$
L^{\otimes(m-1)}=L^{\otimes m} \otimes L^{-1}=L^{\otimes m} \otimes D \otimes B^{-1}=D \otimes E
$$

is also $G$-invariantly effective. It follows that $e_{G}(L)=1$.

Remark 6.1. Let us note the following obvious special case. In the situation of Corollary 6.1 assume in addition that the action of $G$ on $M$ is generically free, so that $K$ is trivial: $K=(e)$. If $L \in \operatorname{Pic}^{G}(M)$ satisfies $\operatorname{vol}_{0}(L)>0$, then $e_{G}(L)=1$.

In view of Remark 6.1 and Corollary 5.3, we obtain:

Corollary 6.4. Suppose that the action of $G$ on $M$ is generically free, and that the hypothesis of Corollary 6.1 are satisfied. If $\operatorname{vol}_{0}(L)>0$, then $\operatorname{vol}_{\mu}\left(L^{\otimes p}\right)=p^{\mathrm{n}-\mathrm{g}} \operatorname{vol}_{\mu}(L)$ for every highest weight $\mu$ and every $p=1,2, \ldots$

Lemma 6.2. Suppose that $B \in \operatorname{Pic}^{G}(M)$ is ample and such that $M^{s}(B) \neq \emptyset$, $\operatorname{codim}\left(M^{\mathrm{u}}(B)\right) \geq 2$. Choose $r \gg 0$ and a very general $\sigma \in H^{0}\left(M, B^{\otimes r e_{G}(B)}\right)^{G}$. Then for every $L \in \operatorname{Pic}^{G}(M)$ and $m \in \mathbb{N}$ we have

$$
h_{\mu}^{0}\left(M,\left(L \otimes B^{-r e_{G}(B)}\right)^{m}\right) \geq h_{\mu}^{0}\left(M, L^{\otimes m}\right)-m h_{\mu}^{0}\left(Z, L^{\otimes m} \otimes \mathcal{O}_{Z}\right),
$$

where $Z=\operatorname{zero}(\sigma)$ is the zero locus of $\sigma$ (here $\left.h_{\mu}^{0}=\operatorname{dim} H_{\mu}^{0}\right)$.

Proof of Lemma 6.2. If $r \gg 0$, we have $\operatorname{Bs}\left(\left|H^{0}\left(M, B^{\otimes r e_{G}(B)}\right)^{G}\right|\right)=$ $M^{\mathrm{u}}(B)$, and by assumption the latter has codimension $\geq 2$. Therefore, for general $\sigma_{1}, \ldots, \sigma_{m} \in H^{0}\left(M, B^{\otimes r e_{G}(B)}\right)^{G}$ the zero loci $Z_{j}=\operatorname{zero}\left(\sigma_{j}\right)$ have no irreducible component in common. Hence, tensor power by $\sigma_{1} \otimes \cdots \otimes \sigma_{m}$ induces an exact sequence

$$
0 \rightarrow H^{0}\left(M,\left(L \otimes B^{-r e_{G}(B)}\right)^{m}\right)_{\mu} \rightarrow H^{0}\left(M, L^{\otimes m}\right)_{\mu} \rightarrow \bigoplus_{j=1}^{m} H^{0}\left(Z_{j}, L^{m} \otimes \mathcal{O}_{Z_{j}}\right)_{\mu}
$$

Given a very general $\sigma \in H^{0}\left(M, B^{\otimes r e_{G}(B)}\right)^{G}$, we deduce from this

$$
\begin{aligned}
h_{\mu}^{0}\left(M,\left(L \otimes B^{-r e_{G}(B)}\right)^{m}\right) & \geq h_{\mu}^{0}\left(M, L^{\otimes m}\right)-\sum_{j} h_{\mu}^{0}\left(Z_{j}, L^{\otimes m} \otimes \mathcal{O}_{Z_{j}}\right) \\
& =h_{\mu}^{0}\left(M, L^{\otimes m}\right)-m \cdot h_{\mu}^{0}\left(Z, L^{\otimes m} \otimes \mathcal{O}_{Z}\right)
\end{aligned}
$$

where the latter equality follows from semicontinuity and the very generality of $\sigma$. 
Proposition 6.2. Let $M$ be a complex projective $G$-manifold, and suppose that $B \in \operatorname{Pic}^{G}(M)$ is ample and such that $M^{s}(B) \neq \emptyset, \operatorname{codim}\left(M^{\mathrm{u}}(B)\right) \geq 2$. Then

$$
\limsup _{p \rightarrow+\infty} \frac{\operatorname{vol}_{\mu}\left(L^{\otimes p} \otimes B^{\otimes\left(-r e_{G}(B)\right)}\right)}{p^{\mathrm{n}-\mathrm{g}}}=\operatorname{vol}_{\mu}(L)
$$

for every $L \in \operatorname{Pic}^{G}(M), r=1,2, \ldots$ and maximal weight $\mu$.

Proof. To simplify notation, let us write $C=B^{\otimes r e_{G}(B)}$. We may assume without loss that $r \gg 0$, so that $\operatorname{Bs}\left(H^{0}(M, C)^{G}\right)=\mathrm{M}^{\mathrm{u}}(B)$. Since $C$ is $G$-invariantly effective, by Remark 2.6] we have

$$
\left.\operatorname{vol}_{\mu}\left(L^{\otimes p} \otimes C^{-1}\right)\right) \leq \operatorname{vol}_{\mu}\left(L^{\otimes p}\right) \leq p^{\mathrm{n}-\mathrm{g}} \operatorname{vol}_{\mu}(L)
$$

the second inequality in (25) is immediate from the definition of volume.

On the other hand, if $Z=\operatorname{zero}(\sigma)$ for some very general $\sigma \in H^{0}(M, C)^{G}$, by Lemma 6.2 applied with $L$ replaced by $L^{\otimes p}$ we have

$$
h_{\mu}^{0}\left(M,\left(L^{\otimes p} \otimes C^{-1}\right)^{\otimes m}\right) \geq h_{\mu}^{0}\left(M, L^{\otimes p m}\right)-m h_{\mu}^{0}\left(Z, L^{\otimes p m} \otimes \mathcal{O}_{Z}\right) .
$$

Dividing by $m^{\mathrm{n}-\mathrm{g}} /(\mathrm{n}-\mathrm{g})$ ! and taking the limsup, we obtain:

$$
\operatorname{vol}_{\mu}\left(M, L^{\otimes p} \otimes C^{-1}\right) \geq \operatorname{vol}_{\mu}\left(M, L^{\otimes p}\right)-(\mathrm{n}-\mathrm{g}) \operatorname{vol}_{\mu}\left(Z, L^{\otimes p} \otimes \mathcal{O}_{Z}\right)
$$

Now we remark that $\operatorname{vol}_{\mu}\left(Z, L^{\otimes p} \otimes \mathcal{O}_{Z}\right) \leq p^{\mathrm{n}-\mathrm{g}-1} \operatorname{vol}_{\mu}\left(Z, L \otimes \mathcal{O}_{Z}\right)$, and that the latter volume is finite by Proposition 4.4. Therefore, dividing by $p^{\mathrm{n}-\mathrm{g}}$ and taking the limsup, we obtain:

$$
\limsup _{p \rightarrow+\infty} \frac{\operatorname{vol}_{\mu}\left(M, L^{\otimes p} \otimes C^{-1}\right)}{p^{\mathrm{n}-\mathrm{g}}} \geq \limsup _{p \rightarrow+\infty} \frac{\operatorname{vol}_{\mu}\left(M, L^{\otimes p}\right)}{p^{\mathrm{n}-\mathrm{g}}} .
$$

The statement of Proposition 6.2 is now a consequence of Proposition 5.1

If in addition $B \in \operatorname{Pic}^{G}(M)$ as in the hypothesis of Proposition 6.2 can be chosen regular, a stronger statement holds:

Corollary 6.5. Suppose that there exists $B \in \operatorname{Pic}^{G}(M)$ ample and regular and such that $\operatorname{codim}\left(M^{\mathrm{u}}(B)\right) \geq 2$. Let $A \in \operatorname{Pic}^{G}(M)$ be any ample $G$ linearized line bundle with $M^{\mathrm{S}}(A) \neq \emptyset$. Suppose $r \gg 0$. Then

$$
\limsup _{p \rightarrow+\infty} \frac{\operatorname{vol}_{\mu}\left(L^{\otimes p} \otimes A^{\otimes\left(-r e_{G}(A)\right)}\right)}{p^{\mathrm{n}-\mathrm{g}}}=\operatorname{vol}_{\mu}(L)
$$

for every $L \in \operatorname{Pic}^{G}(M)$ and maximal weight $\mu$. 
Proof. One inequality is obvious, as in the proof of Proposition 6.2 In the opposite direction, let us choose $s \gg 0$ so that the numerical equivalence class of $H=A \otimes B^{\otimes s e_{G}(B)}$ lies in the interior of the same chamber as the class of $B$; in particular, $H$ is ample and regular, and $\operatorname{codim}\left(M^{\mathrm{u}}(H)\right) \geq 2$. We may assume that $B^{\otimes n s e_{G}(B)}$ is effective for every integer $n \gg 0$. We have $e_{G}(H)=e_{G}(A)$. Therefore, setting $r^{\prime}=\operatorname{rse}_{G}(B)$, we have:

$$
\begin{aligned}
\limsup _{p \rightarrow+\infty} \frac{\operatorname{vol}_{\mu}\left(L^{\otimes p} \otimes A^{-r e_{G}(A)}\right)}{p^{\mathrm{n}-\mathrm{g}}} & \geq \limsup _{p \rightarrow+\infty} \frac{\operatorname{vol}_{\mu}\left(L^{\otimes p} \otimes\left(A \otimes B^{\otimes e_{G}(B)}\right)^{-r e_{G}(A)}\right)}{p^{\mathrm{n}-\mathrm{g}}} \\
& =\limsup _{p \rightarrow+\infty} \frac{\operatorname{vol}_{\mu}\left(L^{\otimes p} \otimes H^{-r^{\prime} e_{G}(H)}\right)}{p^{\mathrm{n}-\mathrm{g}}} \\
& =\operatorname{vol}_{\mu}(L) .
\end{aligned}
$$

The last equality holds by Proposition 6.2.

Remark 6.2. In view of Proposition 5.1, one can actually strengthen the statement of Proposition 6.2 (and Corollary 6.5) as follows: let $p_{i} \uparrow+\infty$ be a sequence of positive integers prime with $e_{G}(L)$. Then

$$
\lim _{i \rightarrow+\infty} \frac{\operatorname{vol}_{\mu}\left(L^{\otimes p_{i}} \otimes B^{\otimes\left(-r e_{G}(B)\right)}\right)}{p_{i}^{\mathrm{n}-\mathrm{g}}}=\operatorname{vol}_{\mu}(L) .
$$

The equivariant Kodaira Lemma (Corollary 6.1) implies a characterization of nef and big line bundles with positive 0-volume.

Corollary 6.6. Suppose that there exists $B \in \mathrm{Pic}^{G}(M)$ ample and such that $M^{s}(B) \neq \emptyset, \operatorname{codim}\left(M^{\mathrm{u}}(B)\right) \geq 2$. Then the following conditions are equivalent on $L \in \operatorname{Pic}^{G}(M)$ :

1. $L$ is nef and big, and $\operatorname{vol}_{0}(L)>0$;

2. there exists $E \in \operatorname{Pic}^{G}(M)$ which is $G$-invariantly effective and is such that $L^{\otimes k} \otimes E^{-1} \in \mathrm{Pic}^{G}(M)$ is ample and has non-empty stable locus, for every $k \gg 0$.

Proof. 1. implies 2.: Perhaps after replacing $B$ by some power $B^{\otimes r e_{G}(B)}$, $r \gg 0$, we may assume that $H^{0}(M, B)^{G}$ globally generates $B$ on the dense open subset $M^{s s}(B)$. By Corollary 6.1, we may find $k_{0} \gg 0$ and a $G$ invariantly effective $E \in \operatorname{Pic}^{G}(M)$ such that $L^{\otimes k_{0}}=B \otimes E$. Suppose $k \gg 0$ and $H^{0}\left(M, L^{\otimes k}\right)^{G} \neq\{0\}$, and choose $\tau \in H^{0}\left(M, L^{\otimes k}\right)^{G}, \tau \neq 0$. Let $U_{\tau}=$ $\{p \in M: \tau(p) \neq 0\}$ and choose $p \in U_{\tau} \cap M^{\mathrm{s}}(B)$ : in particular, $p$ has finite stabilizer $\widetilde{G}_{p} \subseteq \widetilde{G}$. We can find $\sigma \in H^{0}(M, B)^{G}$ such that $\sigma(p) \neq 0$ and the $\widetilde{G}$-orbit of $p$ is closed in $U_{\sigma}=\{p: \sigma(p) \neq 0\}$. Then clearly $\tau \otimes \sigma(p) \neq 0$ and 
$\widetilde{G} \cdot p$ is closed in $U_{\tau \otimes \sigma} \subseteq U_{\sigma}$. Thus $L^{\otimes k} \otimes B$ is ample and $p \in M^{\mathrm{s}}\left(L^{\otimes k} \otimes B\right)$. Now $L^{\otimes\left(k+k_{0}\right)}=\left(L^{\otimes k} \otimes B\right) \otimes E$.

2. implies 1.: obvious.

\section{Relation to numerical equivalence}

We shall now show that, at least under certain hypothesis, the equivariant volumes $\operatorname{vol}_{\mu}(L)$ depend only on the numerical equivalence class of $L \in$ $\operatorname{Pic}^{G}(M)$.

Referring to Definition 2.1 and Remark 2.1, we have:

Lemma 7.1. Suppose $B \in \operatorname{Pic}^{G}(M)$ is ample and $M^{\mathrm{s}}(B) \neq \emptyset$. Then there exist arbitrarily large integers $r \in \mathbb{N}$ such that

$$
H^{0}\left(M, B^{\otimes r e_{G}(B)} \otimes P\right)^{G} \neq\{0\},
$$

for every $P \in \operatorname{Pic}^{G}(M)_{0}^{\prime}$.

Proof of Lemma 7.1. To begin with, let us remark that if $f: \widetilde{M} \rightarrow M$ is an equivariant projective morphism, then the pull-back $f^{*}: \operatorname{Pic}^{G}(M) \rightarrow$ $\operatorname{Pic}^{G}(\widetilde{M})$ satisfies

$$
f^{*}\left(\operatorname{Pic}^{G}(M)_{0}\right) \subseteq \operatorname{Pic}^{G}(\widetilde{M})_{0}, f^{*}\left(\operatorname{Pic}^{G}(M)_{0}^{\prime}\right) \subseteq \operatorname{Pic}^{G}(\widetilde{M})_{0}^{\prime} .
$$

Thus, without loss of generality we may pass if necessary to a Kirwan resolution of the pair $(M, B)$ so as to assume that $B$ is ample and regular. Let us also replace $B$ by $B^{\otimes e_{G}(B)}$, so as to assume without loss that $e_{G}(B)=1$.

For every $P \in \operatorname{Pic}^{G}(M)_{0}^{\prime}$, the following holds ([DH], Propositions 2.3.3 and 3.1.4):

i): $M^{\mathrm{ss}}(B \otimes P)=M^{\mathrm{ss}}(B) \neq \emptyset$;

ii): $e_{G}(B)=e_{G}(B \otimes P)=1$;

therefore,

iii): there exists $r_{P}$ such that $H^{0}\left(M,(B \otimes P)^{\otimes r}\right)^{G} \neq\{0\}$ for all $r \geq r_{P}$.

Hence, by semicontinuity and compactness, there exists $r_{0}$ such that

$$
H^{0}\left(M, B^{\otimes r} \otimes P^{\otimes r}\right)^{G}=H^{0}\left(M,(B \otimes P)^{\otimes r}\right)^{G} \neq\{0\}
$$

for all $P \in \operatorname{Pic}^{G}(M)_{0}^{\prime}$ and $r \geq r_{0}$. Now suppose that $H^{2}(M, \mathbb{Z})_{\text {tor }} \cong \mathbb{Z}_{a_{1}}^{\oplus m_{1}} \oplus$ $\cdots \oplus \mathbb{Z}_{a_{k}}^{\oplus m_{k}}$ for certain integers $a_{i}, m_{i} \in \mathbb{N}$. If $r \geq r_{0}$ is prime with every $a_{i}$, then $P \mapsto P^{\otimes r}$ is a surjective morphism $\operatorname{Pic}^{G}(M)_{0}^{\prime} \rightarrow \operatorname{Pic}^{G}(M)_{0}^{\prime}$. The statement follows. 
Corollary 7.1. Suppose that there exists an ample $B \in \operatorname{Pic}^{G}(M)$ such that $M^{\mathrm{s}}(B) \neq \emptyset$. If $L, L^{\prime} \in \operatorname{Pic}^{G}(M)$ are numerically equivalent and $\mu$ is a maximal weight, then $\operatorname{vol}_{\mu}(L)>0$ if and only $\operatorname{vol}_{\mu}\left(L^{\prime}\right)>0$.

Proof. Perhaps after passing to a Kirwan resolution, we may assume without loss that $B$ is ample and regular. By Lemma 7.1. perhaps after replacing $B$ by $B^{\otimes r e_{G}(B)}$ for some $r \gg 0$ we may assume without loss that $e_{G}(B)=1$ and that $H^{0}(M, B \otimes P)^{G} \neq\{0\}$ for every $P \in \operatorname{Pic}^{G}(M)_{0}^{\prime}$.

Suppose $\operatorname{vol}_{\mu}(L)>0$. By Corollary 6.1, there exists a sequence $k_{i} \in$ $\mathbb{N}_{\mu}(L)$ with $k_{i} \uparrow+\infty$ such that $L^{\otimes k_{i}}=B \otimes F_{i}$, with $\operatorname{dim} H^{0}\left(M, F_{i}\right)_{\mu}=$ $O\left(k_{i}^{\mathrm{n}-\mathrm{g}}\right)$ as $i \rightarrow+\infty$.

Since $L \sim_{\mathrm{n}} L^{\prime}$, we have $L^{\prime}=L \otimes P$ for some $P \in \operatorname{Pic}^{G}(M)_{0}^{\prime}$. Thus, $\left(L^{\prime}\right)^{\otimes k_{i}}=\left(B \otimes P^{\otimes k_{i}}\right) \otimes F_{i}$. Now $H^{0}\left(M, B \otimes P^{\otimes k_{i}}\right)^{G} \neq\{0\}$, and therefore $\operatorname{dim} H^{0}\left(M, F_{i}\right)_{\mu} \leq \operatorname{dim} H^{0}\left(M,\left(L^{\prime}\right)^{\otimes k_{i}}\right)_{\mu}$. Consequently, $\operatorname{dim} H^{0}\left(M,\left(L^{\prime}\right)^{\otimes k_{i}}\right)_{\mu}=$ $O\left(k_{i}^{\mathrm{n}-\mathrm{g}}\right)$, so that $\operatorname{vol}_{\mu}\left(L^{\prime}\right)>0$.

Theorem 7.1. Let us suppose that there exists $B \in \operatorname{Pic}^{G}(M)$ ample and such that $M^{\mathrm{s}}(B) \neq \emptyset$, $\operatorname{codim}\left(M^{\mathrm{u}}(B)\right) \geq 2$. If $L, L^{\prime} \in \operatorname{Pic}^{G}(M), L \sim_{\mathrm{n}} L^{\prime}$ and $\mu$ is a maximal weight, then

$$
\operatorname{vol}_{\mu}(L)=\operatorname{vol}_{\mu}\left(L^{\prime}\right) .
$$

Proof of Theorem 7.1. By assumption, $L^{\prime}=L \otimes P$ for some $P \in$ $\operatorname{Pic}^{G}(M)_{0}^{\prime}$. Given Corollary 7.1, we may assume that $\operatorname{vol}_{\mu}(L)>0$. In view of Lemma 7.1] there exists $r \in \mathbb{N}$ such that $H^{0}\left(M, B^{\otimes r e_{G}(B)} \otimes P^{-p}\right)^{G} \neq\{0\}$ for every $p \in \mathbb{Z}$. Recalling Remark [2.6, we then have

$$
\operatorname{vol}_{\mu}\left((L \otimes P)^{\otimes p} \otimes B^{-\otimes r e_{G}(B)}\right) \leq \operatorname{vol}_{\mu}\left(L^{\otimes p}\right) \leq p^{\mathrm{n}-\mathrm{g}} \operatorname{vol}_{\mu}(L),
$$

for any maximal weight $\mu$. Proposition 6.2, applied with $L$ replaced by $L \otimes P$, now implies $\operatorname{vol}_{\mu}(L \otimes P) \leq \operatorname{vol}_{\mu}(L)$. The statement then follows by exchanging the roles of $P$ and $P^{-1}$.

Remark 7.1. Given Theorem 7.1, for every maximal weight $\mu$ there is a welldefined function $\operatorname{vol}_{\mu}: \operatorname{Num}^{G}(M) \rightarrow \mathbb{R}$. In view of Example 3.1 this does not extend to a well-defined function on $\operatorname{Num}^{G}(M)_{\mathbb{Q}}=: \operatorname{Num}^{G}(M) \otimes \mathbb{Q}$ in a natural way, except in the notable case $\mu=0$. In fact, momentarily adopting additive notation, given any $P \in \mathrm{NS}^{G}(M)_{\mathbb{Q}}$, there exists $a \in \mathbb{N}$ such that $a P \in \mathrm{NS}^{G}(M)$; we may then let $\operatorname{vol}_{0}(P)=: \frac{1}{a^{\mathrm{n}-\mathrm{g}}} \operatorname{vol}_{0}(a P)$. This is well-defined (that is, independent of the choice of $a$ ) by Corollary 5.4. 


\section{The $G$-big cone}

Recall that $\mathrm{C}^{G}(M) \subseteq \mathrm{NS}^{G}(M)_{\mathbb{R}}$ is the $G$-ample cone of [DH]. The integral points in its interior Int $\left(\mathrm{C}^{G}(M)\right)$ are the numerical equivalence classes of the ample $L \in \operatorname{Pic}^{G}(M)$ such that $M^{\mathrm{s}}(L) \neq \emptyset$ (the non-rational points $\xi \in$ Int $\left(\mathrm{C}^{G}(M)\right)$ have a similar interpretation in terms of Kähler classes [DH] $)$. Thus, in the situation of Corollary 8.1 below, Int $\left(\mathrm{C}^{G}(M)\right)$ is a non-empty open cone in $\mathrm{NS}^{G}(M)_{\mathbb{R}}$.

In the special case $\mu=0$, Corollary 6.1 yields the following characterization:

Corollary 8.1. Let $M$ be a complex projective $G$-manifold, and suppose that there exists an ample $B \in \operatorname{Pic}^{G}(M)$ such that $M^{\mathrm{s}}(B) \neq \emptyset, \operatorname{codim}\left(M^{\mathrm{u}}(B)\right) \geq$

2. Then the following conditions on $L \in \operatorname{Pic}^{G}(M)$ are equivalent:

i): $\operatorname{vol}_{0}(L)>0$;

ii): there exist $k \in \mathbb{N}$ and $F \in \operatorname{Pic}^{G}(M)$ with $H^{0}(M, F)^{G} \neq\{0\}$ such that $L^{\otimes k}=B^{e_{G}(B)} \otimes F ;$

iii): there exist $k \in \mathbb{N}, A, F \in \operatorname{Pic}^{G}(M)$ with $A \in \operatorname{Int}\left(\mathrm{C}^{G}(M)\right), H^{0}(M, F)^{G} \neq$ $\{0\}$, such that $L^{\otimes k}=B^{e_{G}(B)} \otimes F$.

Definition 8.1. i): An element $\xi \in \mathrm{NS}^{G}(M)_{\mathbb{R}}$ will be called $G$-big if it has the form $\xi=\sum_{i=1}^{r} a_{i}\left[L_{i}\right]$, where $a_{i}>0$ and $L_{i} \in \operatorname{Pic}^{G}(M)$ satisfies $\operatorname{vol}_{0}\left(L_{i}\right)>0$ for every $i$. The $G$-big cone $\operatorname{Big}^{G}(M) \subseteq \mathrm{NS}^{G}(M)_{\mathbb{R}}$ is the convex cone of all $G$-big elements of $\operatorname{NS}^{G}(M)_{\mathbb{R}}$.

ii): An element $\xi \in \mathrm{NS}^{G}(M)_{\mathbb{R}}$ will be called $G$-effective if it has the form $\xi=\sum_{i=1}^{r} a_{i}\left[L_{i}\right]$, where $a_{i}>0$ and $L_{i} \in \operatorname{Pic}^{G}(M)$ satisfies $M^{\text {ss }}\left(L_{i}\right) \neq \emptyset$ for every $i$. The $G$-effective cone $\operatorname{Eff}^{G}(M) \subseteq \mathrm{NS}^{G}(M)_{\mathbb{R}}$ is the convex cone of all $G$-effective elements of $\mathrm{NS}^{G}(M)_{\mathbb{R}}$.

iii): The G-pseudo-effective cone is the closure $\overline{\operatorname{Eff}^{G}(M)} \subseteq \mathrm{NS}^{G}(M)_{\mathbb{R}}$.

By analogy with Proposition 2.2.22 of $[\mathrm{L}$, and essentially by the same argument, we have:

Proposition 8.1. In the situation of Corollary [8.1, $\xi \in \mathrm{NS}^{G}(M)_{\mathbb{R}}$ is $G$-big if and only if $\xi=a+b$ for some $a \in \operatorname{Int}\left(\mathrm{C}^{G}(M)\right)$ and $b \in \operatorname{Eff}^{G}(M)$.

This leads to the following analogue of Theorem 2.2.26 of $[\mathrm{L}$ (the proof is similar): 
Theorem 8.1. Let $M$ be a complex projective $G$-manifold, and suppose that there exists an ample $B \in \mathrm{Pic}^{G}(M)$ such that $M^{\mathrm{s}}(B) \neq \emptyset, \operatorname{codim}\left(M^{\mathrm{u}}(B)\right) \geq$ 2. Then the $G$-big cone is open. Furthermore, $\operatorname{Big}^{G}(M)$ is the interior of the $G$-pseudoeffective cone, and the G-pseudoeffective cone is the closure of the G-big cone:

$$
\operatorname{Big}^{G}(M)=\operatorname{Int}\left(\overline{\operatorname{Eff}^{G}(M)}\right), \quad \overline{\operatorname{Eff}^{G}(M)}=\overline{\operatorname{Big}^{G}(M)}
$$

Remark 8.1. Let us take up again the special case of generically free actions, under hypothesis of Corollary 6.1. By Corollary 6.4 and the argument used in Remark 17.1, for every $\mu$ the function $\operatorname{vol}_{\mu}$ extends to a well defined homogeneous function of degree $\mathrm{n}-\mathrm{g}$ on the set $\operatorname{Big}^{G}(M)_{\mathbb{Q}} \subseteq \operatorname{Big}^{G}(M)$ of rational points in the $G$-big cone. We shall denote this function by $\operatorname{vol}_{\mu}: \operatorname{Big}^{G}(M)_{\mathbb{Q}} \rightarrow \mathbb{R}$.

\section{Continuity}

We have noted in Remark 7.1 that the functions $\operatorname{vol}_{\mu}: \operatorname{Pic}^{G}(M) \rightarrow \mathbb{R}$ descend to well-defined functions on $\mathrm{Num}^{G}(M)$, and that by homogeneity $\mathrm{vol}_{0}$ extends to a function $\operatorname{vol}_{0}: \operatorname{Num}^{G}(M)_{\mathbb{Q}} \rightarrow \mathbb{R}$ homogeneous of degree $\mathrm{n}-\mathrm{g}$. We have also noted in Remark 8.1 that, if the action of $G$ on $M$ is generically free, then $\operatorname{vol}_{\mu}$ extends to a function on $\operatorname{Big}^{G}(M)_{\mathbb{Q}}$ for every $\mu$, also homogeneous of degree $n-g$.

We shall now dwell on the continuity properties of these functions and prove the estimates (26) and (27) below. We shall deduce that, at least under appropriate hypothesis, $\mathrm{vol}_{0}$ extends to a continuous real-valued function on $\mathrm{NS}^{G}(M)_{\mathbb{R}}=: \operatorname{Num}^{G}(M)_{\mathbb{Q}} \otimes \mathbb{R}$, and that $\operatorname{vol}_{\mu}$ extends to a continuous realvalued function on $\operatorname{Big}^{G}(M) \subseteq \mathrm{NS}^{G}(M)_{\mathbb{R}}$.

With some abuse, we shall not distinguish notationally an element $A \in$ $\operatorname{Pic}^{G}(M)$ from its equivalence class in $\operatorname{Num}^{G}(M)_{\mathbb{Q}} \subseteq \mathrm{NS}^{G}(M)_{\mathbb{R}}$.

The content of this section is given by the following two Theorems:

Theorem 9.1. Let $M$ be a complex projective $G$-manifold. Suppose that there exists $B \in \mathrm{Pic}^{G}(M)$ ample and regular, satisfying $\operatorname{codim}\left(M^{\mathrm{u}}(B)\right) \geq 2$. Let us fix any norm on $\mathrm{NS}^{G}(M)_{\mathbb{R}}$. Then there exists $C>0$ such that for any $D, D^{\prime} \in \mathrm{NS}^{G}(M)_{\mathbb{Q}}$ we have:

$$
\left|\operatorname{vol}_{0}(D)-\operatorname{vol}_{0}\left(D^{\prime}\right)\right| \leq C \max \left\{\|D\|,\left\|D^{\prime}\right\|\right\}^{\mathrm{n}-\mathrm{g}-1} \cdot\left\|D-D^{\prime}\right\| .
$$

Theorem 9.2. In the situation of Theorem 9.1, assume in addition that the action of $G$ on $M$ is generically free. Then there exists $C>0$ such that for 
any $D, D^{\prime} \in \operatorname{Big}^{G}(M)_{\mathbb{Q}}$ and every highest weight $\mu$ we have:

$$
\left|\operatorname{vol}_{\mu}(D)-\operatorname{vol}_{\mu}\left(D^{\prime}\right)\right| \leq C \operatorname{dim}\left(V_{\mu}\right)^{2} \max \left\{\|D\|,\left\|D^{\prime}\right\|\right\}^{\mathrm{n}-\mathrm{g}-1} \cdot\left\|D-D^{\prime}\right\| .
$$

We shall give an explicit proof of Theorem 9.1 and leave it to the reader to make the necessary changes for the proof of Theorem 9.2. The statement and the proof of Theorem 9.1 are inspired by Theorem 2.2.44 of [L]. We shall use throughout additive notation.

Proof. By assumption, there exists an open (conic) chamber $C$ in the $G$-ample cone $C^{G}(M) \subseteq \mathrm{NS}^{G}(M)_{\mathbb{R}}$ such that $B \in C$ and every $A \in \operatorname{Pic}^{G}(M)$ whose numerical class is in $C$ satisfies $M^{\mathrm{s}}(A)=M^{\mathrm{ss}}(A)=M^{\mathrm{s}}(B) \neq \emptyset$. Setting $r=: \operatorname{dim} \mathrm{NS}^{G}(M)_{\mathbb{R}}$, we can then find $A_{1}, \ldots, A_{r} \in \operatorname{Pic}^{G}(M)$ whose numerical classes all belong to $C$, and such that $\mathcal{B}=\left\{A_{1}, \ldots, A_{r}\right\}$ is a basis of $\mathrm{NS}^{G}(M)_{\mathbb{R}}$. Perhaps after replacing each $A_{j}$ by a sufficiently large multiple we may, and shall, suppose that the $A_{j}$ 's are all very ample and descend to very ample line bundles on the GIT quotient $M_{0}\left(A_{j}\right)=M_{0}(B)$.

As in $\left[\mathrm{L}\right.$, we shall use the norm $\|\xi\|=\max \left\{\left|x_{i}\right|\right\}$, where $\xi=\sum_{i} x_{i} \cdot A_{i}$.

Proposition 9.1. There exists $C>0$ such that for any $D, D^{\prime} \in \mathrm{NS}^{G}(M)$ of the form

$$
\begin{aligned}
D & =a_{1} \cdot A_{1}+\ldots+a_{r} \cdot A_{r} \\
D^{\prime} & =a_{1}^{\prime} \cdot A_{1}+\ldots+a_{r}^{\prime} \cdot A_{r},
\end{aligned}
$$

where $a_{i}, a_{i}^{\prime} \in \mathbb{Z}$ for every $i$, we have:

$$
\left|\operatorname{vol}_{0}(D)-\operatorname{vol}_{0}\left(D^{\prime}\right)\right| \leq C \max \left\{\|D\|,\left\|D^{\prime}\right\|\right\}^{\mathrm{n}-\mathrm{g}-1} \cdot\left\|D-D^{\prime}\right\| .
$$

Proof of Proposition 9.1. By abuse of language, it will be convenient to identify each $A_{j}$ with some general divisor in the linear series $\left|A_{j}\right|^{G}$. With this interpretation, every $A_{j}$ is a $G$-invariantly effective divisor in $M$, nonsingular away from $M^{\mathrm{u}}(B)$. We shall assume to begin with that $a_{i} \geq a_{i}^{\prime}$ for every $i$; thus $b_{i}=: a_{i}-a_{i}^{\prime} \geq 0$ for every $i$ and $D^{\prime}=D-B$, where $B=\sum_{i} b_{i} \cdot A_{i}$ is $G$-invariantly effective. Thus, $\operatorname{vol}_{0}(D-B) \leq \operatorname{vol}_{0}(D)$.

In order to obtain a bound in the opposite direction, let us choose for every $j$ a very general $E_{j} \in\left|A_{j}\right|^{G}$. Then $E_{j}$ is $G$-invariantly effective, reduced, and meets every $A_{i}$ properly. Let us set $D_{j}=\left.D\right|_{E_{j}}$. By Proposition 4.4. $\operatorname{vol}_{0}\left(E_{j}, D_{j}\right)<+\infty$.

Lemma 9.1. Given that $b_{i} \geq 0$ for every $i$, we have

$$
\operatorname{vol}_{0}(D-B) \geq \operatorname{vol}_{0}(D)-(n-g) \cdot \sum_{j} b_{j} \cdot \operatorname{vol}_{0}\left(E_{j}, D_{j}\right) .
$$


Proof of Lemma 9.1. It suffices by induction to consider the case $B=$ $b_{1} A_{1}$.

For $m \gg 0$, let us choose $m b_{1}$ very general divisors $F_{\alpha} \in\left|A_{1}\right|^{G}$. This yields an equivariant exact sequence

$$
0 \rightarrow \mathcal{O}_{M}\left(m D-m b_{1} A_{1}\right) \rightarrow \mathcal{O}_{M}(m D) \rightarrow \bigoplus_{\alpha=1}^{m b_{1}} \mathcal{O}_{F_{\alpha}}(m D)
$$

By the very generality of our choices, we have an estimate

$$
\begin{aligned}
\operatorname{dim} H^{0}\left(F_{\alpha}, \mathcal{O}_{F_{\alpha}}(m D)\right)^{G} & =\operatorname{dim} H^{0}\left(E_{1}, \mathcal{O}_{E_{1}}\left(m D_{1}\right)\right)^{G} \\
& \leq \operatorname{vol}_{0}\left(E_{1}, D_{1}\right) \cdot \frac{m^{\mathrm{n}-\mathrm{g}-1}}{(\mathrm{n}-\mathrm{g}-1) !}+o\left(m^{\mathrm{n}-\mathrm{g}-1}\right) .
\end{aligned}
$$

Thus, setting $h^{0}(M, L)_{0}=: \operatorname{dim} H^{0}(M, L)^{G}$ for $L \in \operatorname{Pic}^{G}(M)$,

$$
\begin{aligned}
\frac{(\mathrm{n}-\mathrm{g}) !}{m^{\mathrm{n}-\mathrm{g}}} h^{0}\left(M, m\left(D-b_{1} A_{1}\right)\right)_{0} \geq & \frac{(\mathrm{n}-\mathrm{g}) !}{m^{\mathrm{n}-\mathrm{g}}} h^{0}(M, m D)_{0} \\
& -b_{1}(\mathrm{n}-\mathrm{g}) \frac{(\mathrm{n}-\mathrm{g}-1) !}{m^{\mathrm{n}-\mathrm{g}-1}} h^{0}\left(E_{1}, D_{1}\right)_{0} \\
\geq & \frac{(\mathrm{n}-\mathrm{g}) !}{m^{\mathrm{n}-\mathrm{g}}} h^{0}(M, m D)_{0} \\
& -(\mathrm{n}-\mathrm{g}) b_{1} \operatorname{vol}_{0}\left(E_{1}, D_{1}\right)+o(1) .
\end{aligned}
$$

and taking limsup as $m \rightarrow+\infty$ we obtain:

$$
\operatorname{vol}_{0}\left(\mathcal{O}_{M}\left(D-b_{1} A_{1}\right)\right) \geq \operatorname{vol}_{0}\left(\mathcal{O}_{M}(D)\right)-b_{1}(\mathrm{n}-\mathrm{g}) \operatorname{vol}_{0}\left(E_{1}, D_{1}\right) .
$$

This completes the proof of Lemma 9.1

Proposition 9.2. There exists $C>0$ such that

$$
\operatorname{vol}_{0}\left(E_{j}, D_{j}\right) \leq C\|D\|^{\mathrm{n}-\mathrm{g}-1}
$$

for every $j=1, \ldots, r$.

Proof of Proposition 9.2. Let $E_{j}=\sum_{k=1}^{r_{j}} E_{j k}$ be the decomposition of $E_{j}$ into its irreducible components, and for every $j$ and $k$ let us set $D_{j k}=:\left.D\right|_{E_{j k}}$ (notice that $E_{j}$ may be assumed irreducible as soon as $\mathrm{n}-\mathrm{g}>1$ ). Since $\operatorname{vol}_{\mu}\left(E_{j}, D_{j}\right) \leq \sum_{k=1}^{r_{j}} \operatorname{vol}_{\mu}\left(E_{j k}, D_{j k}\right)$, it suffices to prove the statement for each irreducible component $E_{j k}$. Let us set

$$
D^{+}=: \sum_{i=1}^{r}\left|a_{i}\right| D_{i}, \quad D_{j}^{+}=:\left.D^{+}\right|_{E_{j}}
$$


Then

$$
D_{j k}^{+}-D_{j k}=\left.\sum_{i}\left(\left|a_{i}\right|-a_{i}\right) A_{i}\right|_{E_{j k}}
$$

where each $\left.A_{i}\right|_{E_{j k}}$ is a $G$-invariantly effective line bundle on $E_{j k}$. It follows by Remark 2.6 that

$$
\operatorname{vol}_{0}\left(E_{j k}, D_{j k}\right) \leq \operatorname{vol}_{0}\left(E_{j k}, D_{j k}^{+}\right)
$$

Since $\|D\|=\left\|D^{+}\right\|$, it thus suffices to prove the statement of Proposition 9.2 using $D^{+}$in place of $D$. To this end, let us first record the following:

Lemma 9.2. There exists $R \in \mathrm{GL}\left(\mathrm{NS}^{G}(M)_{\mathbb{R}}\right)$ with the following properties:

i): for every $i=1, \ldots, r, R\left(A_{i}\right)-A_{i}$ represents a $G$-invariantly effective positive power of $B$;

ii): for every $i=1, \ldots, r, R\left(A_{i}\right)$ lies in the interior of the chamber $C$.

In particular, $R\left(D^{+}\right)$also lies in the interior of the chamber $C$.

Proof of Lemma 9.2. We need only define $R\left(A_{i}\right)=: A_{i}+r e_{G}(B) B$ for some $r \gg 0$, and then extend by linearity. This establishes i), and ii) is a consequence of i) given our choice of the $A_{i}$ 's.

Back to the proof of Proposition 9.2, it follows from Lemma 9.2 that $R\left(D^{+}\right)-D^{+}$is $G$-invariantly effective, and that so is its restriction to $E_{j}$ for every $j$. Therefore,

$$
\operatorname{vol}_{0}\left(E_{j k}, D_{j k}\right) \leq \operatorname{vol}_{0}\left(E_{j k}, D_{j k}^{+}\right) \leq \operatorname{vol}_{0}\left(E_{j k},\left.R\left(D^{+}\right)\right|_{E_{j k}}\right)
$$

To fix ideas, let us first suppose that all the $E_{j k}$ 's are non-singular. If $M_{0}(B)$ is the GIT quotient of $M$ with respect to the linearization $A_{j}$, the GIT quotient of $E_{j k} \subseteq M$ with respect to the same linearization is an ample divisor $\left(E_{j k}\right)_{0} \subseteq M_{0}(B)$; hence it is $(\mathrm{n}-\mathrm{g}-1)$-dimensional .

Since $\left.R\left(D^{+}\right)\right|_{E_{j k}}$ is an ample and regular $G$-linearized line bundle, by Corollary 3.2 and Remark 3.2 we have

$$
\begin{aligned}
\operatorname{vol}_{0}\left(E_{j k},\left.R\left(D^{+}\right)\right|_{E_{j k}}\right) & =\operatorname{vol}_{0}\left(\left(E_{j k}\right)_{0}, \Omega_{R\left(D^{+}\right)}^{(j k)}\right) \\
& =C_{j k}^{\prime} \int_{\left(E_{j k}\right)_{0}}\left(\Omega_{R\left(D^{+}\right)}^{(j k)}\right)^{\mathrm{n}-\mathrm{g}-1} \\
& \leq C_{j k}^{\prime \prime}\left\|D^{+}\right\|^{\mathrm{n}-\mathrm{g}-1},
\end{aligned}
$$


where $\Omega_{R\left(D^{+}\right)}^{(j k)}$ is a Kähler form on $\left(E_{j}\right)_{0}$, which we may choose to depend linearly on $R\left(D^{+}\right)$, whence on $D^{+}\left(\Omega_{R\left(D^{+}\right)}^{(j k)}\right.$ is the restriction to $\left(E_{j k}\right)_{0} \subseteq$ $M_{0}(B)$ of a Kähler form on $M_{0}(B)$ of the form $\sum_{i} \ell_{i} \Omega_{A_{i}}$, where $R\left(D^{+}\right)=$ $\left.\sum_{i} \ell_{i} A_{i}\right)$.

Let us now argue in general.

Lemma 9.3. Given the generality in its choice, $E_{j k}$ satisfies the following properties:

i): $E_{j k}$ is non-singular away from $M^{\mathrm{u}}\left(A_{j}\right)=M^{\mathrm{u}}\left(R\left(D^{+}\right)\right)=M^{\mathrm{u}}(B)$;

ii): if $\Phi_{R\left(D^{+}\right)}: M \rightarrow \mathfrak{g}^{*}$ is the moment map of $R\left(D^{+}\right), E_{j k}$ is non-singular in a neighbourhood of $E_{j k} \cap \Phi_{R\left(D^{+}\right)}^{-1}(0)$;

iii): $E_{j k}$ is transversal to $\Phi_{R\left(D^{+}\right)}^{-1}(0)$.

Proof of Lemma 9.3. i) follows directly from Bertini's Theorem and the definition of unstable locus.

Given i), ii) follows from the well-known relation between the semi-stable locus and the zero locus of the moment map Ki1.

As to iii), by the arguments of Lemma 3 in [P2] and compactness one can see the following: There exist a finite number of holomorphic embeddings $\varphi_{i}: B_{\mathrm{n}-\mathrm{g}}(0,1) \rightarrow M$, where $B_{\mathrm{n}-\mathrm{g}}(0,1) \subseteq \mathbb{C}^{\mathrm{n}-\mathrm{g}}$ is the unit ball centered at the origin, satisfying a): $\varphi_{i}\left(B_{\mathrm{n}-\mathrm{g}}(0,1)\right) \subseteq \Phi_{R\left(D^{+}\right)}^{-1}(0)$; b): as a submanifold of $\Phi_{R\left(D^{+}\right)}^{-1}(0), \varphi_{i}\left(B_{\mathrm{n}-\mathrm{g}}(0,1)\right)$ is transversal to every $G$-orbit; c): the union $\bigcup_{i} \varphi_{i}\left(B_{\mathrm{n}-\mathrm{g}}(0,1)\right)$ maps surjectively onto $M_{0}\left(R\left(D^{+}\right)\right)=M_{0}(B)$. In view the local analytic proof of Bertini's theorem in $\mathrm{GH}$, we may assume that $E_{j}$ is transversal to each $\varphi_{i}(B)$. By $G$-invariance, it is then transversal to all of $\Phi_{R\left(D^{+}\right)}^{-1}(0)$.

This completes the proof of Lemma 9.3 .

Let $f_{j k}: \widetilde{E}_{j k} \rightarrow E_{j k}$ be a $G$-equivariant resolution of singularities, which may be assumed to be an isomorphism away from $M^{\mathrm{u}}\left(R\left(D^{+}\right)\right)=M^{\mathrm{u}}(B)$.

Corollary 9.1. $0 \in \mathfrak{g}^{*}$ is a regular value of the moment map for $f_{j k}^{*}\left(R\left(D^{+}\right)\right)$ given by

$$
\widetilde{\Phi}_{R\left(D^{+}\right)}^{(j k)}=: \Phi_{R\left(D^{+}\right)} \circ f_{j k}: \widetilde{E}_{j k} \longrightarrow \mathfrak{g}^{*},
$$

and $\left(\widetilde{\Phi}_{R\left(D^{+}\right)}^{(j k)}\right)^{-1}(0) \neq \emptyset$. Furthermore, $\widetilde{\Phi}_{R\left(D^{+}\right)}^{(j k)}$ is bounded in norm away from zero on $f_{j k}^{-1}\left(M^{\mathrm{u}}(B)\right)$. 
Proof of Corollary 2.1. The only statement not immediately obvious from Lemma 9.3 is that $\left(\widetilde{\Phi}_{R\left(D^{+}\right)}^{(j k)}\right)^{-1}(0) \neq \emptyset$. To see this, we need only recall that

$$
M^{s}\left(\left.R\left(D^{+}\right)\right|_{E_{j k}}\right)=M^{\mathrm{s}}\left(\left.B\right|_{E_{j k}}\right) \neq \emptyset .
$$

Returning to the proof of Proposition 9.2 , there exists a $G$-invariantly effective exceptional divisor $F_{j k} \subseteq \widetilde{E}_{j k}$ such that $f_{j}^{*}\left(R\left(D^{+}\right)\right)^{\otimes l}\left(-F_{j k}\right) \in$ $\operatorname{Pic}^{G}\left(\widetilde{E}_{j k}\right)$ is a (very) ample $G$-linearized line bundle on $\widetilde{E}_{j k}$, for every $l \gg 0$. Its linearization corresponds to a moment map (with respect to an appropriate Kähler form) of the form

$$
\widetilde{\Phi}_{l j k}=: l \widetilde{\Phi}_{R\left(D^{+}\right)}^{(j)}+\Phi_{F_{j k}}: \widetilde{E}_{j k} \rightarrow \mathfrak{g}^{*},
$$

where $\Phi_{F_{j k}}$ is an appropriate fixed equivariant map. By the above, if $l \gg 0$ then $0 \in \mathfrak{g}^{*}$ is a regular value of $\widetilde{\Phi}_{l j k}$ and $\left(\widetilde{\Phi}_{l j k}\right)^{-1}(0) \neq \emptyset$. Thus,

Lemma 9.4. If $l \gg 0, f_{j}^{*}\left(R_{j}\left(D^{+}\right)\right)^{\otimes l}\left(-F_{j k}\right) \in \operatorname{Pic}^{G}\left(\widetilde{E}_{j k}\right)$ is ample and regular.

Let us then fix $l_{0} \gg 0$ such that $f_{j k}^{*}\left(R\left(D^{+}\right)\right)^{\otimes l_{0}}\left(-F_{j k}\right)$ is ample and regular, and next $a_{0} \gg 0$ such that in addition $f_{j k}^{*}\left(R\left(D^{+}\right)\right)^{\otimes a_{0} l_{0}}\left(-a_{0} F_{j k}\right)$ is $G$-invariantly effective.

Next let $p_{i} \uparrow+\infty$ be a sequence of integers. In view of Corollary [5.4 we have $\operatorname{vol}_{0}\left(\left.R\left(D^{+}\right)\right|_{E_{j k}} ^{\otimes p_{i}}\right)=p_{i}^{\mathrm{n}-\mathrm{g}-1} \operatorname{vol}_{0}\left(\left.R\left(D^{+}\right)\right|_{E_{j}}\right)$ for every $i$. On the upshot, we have

$$
\begin{aligned}
\operatorname{vol}_{0}\left(\left.R\left(D^{+}\right)\right|_{E_{j k}}\right) & \leq \operatorname{vol}_{0}\left(f_{j k}^{*}\left(R\left(D^{+}\right)\right)\right) \\
& =\lim _{i \rightarrow+\infty} \frac{\operatorname{vol}_{0}\left(f_{j k}^{*}\left(R\left(D^{+}\right)\right)^{\otimes p_{i}}\right)}{p_{i}^{\mathrm{n}-\mathrm{g}-1}} \\
& \leq \limsup _{i \rightarrow+\infty} \frac{\operatorname{vol}_{0}\left(f_{j k}^{*}\left(R\left(D^{+}\right)\right)^{\otimes\left(p_{i}+a_{0} l_{0}\right)}\left(-a_{0} F_{j k}\right)\right)}{p_{i}^{\mathrm{n}-\mathrm{g}-1}} .
\end{aligned}
$$

In view of Lemma 9.4 the volume in the last line of (31) may be computed using Theorem 3.1. The Kähler form for $f_{j k}^{*}\left(R\left(D^{+}\right)\right)^{\otimes\left(p_{i}+a_{0} l_{0}\right)}\left(-a_{0} F_{j k}\right)$ may clearly be chosen of the form $\left(p_{i}+a_{0} l_{0}\right) \Omega_{R\left(D^{+}\right)}+\Omega_{F}$, where $\Omega_{R\left(D^{+}\right)}$depends linearly on $R\left(D^{+}\right)$, and $\Omega_{F}$ is fixed. Thus, given (31), we obtain

$$
\begin{aligned}
\operatorname{vol}_{0}\left(\left.R\left(D^{+}\right)\right|_{E_{j k}}\right) & \leq \lim _{i \rightarrow+\infty} \int_{\widetilde{E}_{j k}} \frac{\left(\left(p_{i}+a_{0} l_{0}\right) \Omega_{R\left(D^{+}\right)}+\Omega_{F}\right)^{\mathrm{n}-\mathrm{g}-1}}{p_{i}^{\mathrm{n}-\mathrm{g}-1}} \\
& \leq C\left\|D^{+}\right\|^{\mathrm{n}-\mathrm{g}-1} .
\end{aligned}
$$


Since the $E_{j k}$ 's are in finitely many, the constant $C$ may be chosen independent of $j$ and $k$. The statement of Proposition 9.2 follows.

On the upshot, there exists a constant $C>0$ such that whenever $D=$ $\sum_{i} a_{i} A_{i}$ with $a_{i} \in \mathbb{Z}$ for every $i$, and $B=\sum b_{i} A_{i}$ with $b_{i} \in \mathbb{N}$ for every $i$, we have

$$
\operatorname{vol}_{0}(D)-C\|D\|^{\mathrm{n}-\mathrm{g}-1} \cdot\|B\| \leq \operatorname{vol}_{0}(D-B) \leq \operatorname{vol}_{0}(D) .
$$

Given this, we can now prove (28) without assuming that $B=D-D^{\prime}$ is effective, as follows. Let us write $B=F-E$, where $F=\sum_{i} f_{i} A_{i}$, $E=\sum_{i} e_{i} A_{i}$ and $f_{i}, e_{i} \in \mathbb{N}$ for every $i$. We may also assume that $e_{i} f_{i}=0$ for every $i$. Then (32) implies the inequalities:

$$
\begin{aligned}
\operatorname{vol}_{0}(D)-\operatorname{vol}_{0}(D-F) & \leq C\|D\|^{\mathrm{n}-\mathrm{g}-1} \cdot\|F\| \\
\operatorname{vol}_{0}(D+E-F)-\operatorname{vol}_{0}(D-F) & \leq C\|D+E-F\|^{\mathrm{n}-\mathrm{g}-1} \cdot\|E\| .
\end{aligned}
$$

The statement then follows by the triangle inequality since $\max (\|E\|,\|F\|)=$ $\|E-F\|$. This completes the proof of Proposition 9.1

Back to the proof of Theorem 9.1, now we need only notice that the terms in (28) are all homogeneous of degree $n-g$. Therefore, the inequality in the statement of Theorem 9.1 holds for any $D, D^{\prime} \in \mathrm{NS}^{G}(M)_{\mathbb{Q}}$.

Corollary 9.2. In the hypothesis of Theorem 9.1, $\operatorname{vol}_{0}$ extends to a continuous real-valued function on $\mathrm{NS}^{G}(M)_{\mathbb{R}} \backslash\{0\}$, and on $\mathrm{NS}^{G}(M)_{\mathbb{R}}$ if $\mathrm{n} \geq \mathrm{g}+1$.

Corollary 9.3. In the hypothesis of Theorem 9.2, $\operatorname{vol}_{\mu}$ extends to a continuous real-valued function on $\overline{\operatorname{Big}^{G}(M)} \backslash\{0\}$, and on $\overline{\operatorname{Big}^{G}(M)}$ if $\mathrm{n} \geq \mathrm{g}+1$.

\section{Equivariant Fujita approximations}

In the study of absolute algebro-geometric volumes, an important role is played by Fujita approximations, which can be seen as providing a sort of asymptotic Zariski decomposition of an arbitrary big class $[\mathrm{F}],[\mathrm{DEL}, \mathrm{L}]$. In this section, we shall give an equivariant version of this in the special case of circle actions, under some assumptions on the action of $S^{1}$ on $M$. An equivariant version of Fujita approximations has been given in [P1] for finite group actions.

Theorem 10.1. Let $M$ be a projective $S^{1}$-manifold, and suppose that there exists a regular and ample $B \in \operatorname{Pic}^{S^{1}}(M)$ such that $\operatorname{codim}\left(M^{\mathrm{u}}(B)\right) \geq 2$. Suppose $L \in \operatorname{Pic}^{S^{1}}(M)$ is $S^{1}$-big, and fix $\epsilon>0$. Let $F \subseteq \mathbb{Z}$ be any finite set of weights, with $0 \in F$. Then there exist: 
- an equivariant modification $\beta: \widetilde{M} \rightarrow M$ (depending on $\xi, F$ and $\epsilon$ ), where the $G$-ample cone of $\widetilde{M}$ has non-empty interior,

- $A, E \in \operatorname{Pic}^{S^{1}}(\widetilde{M})$ with $A$ ample and $E S^{1}$-invariantly effective,

- an integer $p>0$ with $\left(p, e_{G}(L)\right)=1$,

such that $\beta^{*}(L)^{\otimes p}=A \otimes E, \operatorname{vol}_{\mu}(A) \geq p^{\mathrm{n}-1}\left(\operatorname{vol}_{\mu}(L)-\epsilon\right)$ for every $\mu \in F$, and $\operatorname{vol}(A) \geq p^{\mathrm{n}}(\operatorname{vol}(L)-\epsilon)$.

Proof. We shall subdivide the proof in a series of Propositions.

Proposition 10.1. A decomposition as in the statement of Theorem 10.1 exists, if we only require $A \in \mathrm{Pic}^{S^{1}}(\widetilde{M})$ to be nef, big and $S^{1}$-big.

Proof. The argument below is inspired by the proof of Theorem 11.4.4 of [L].

Perhaps after replacing $B$ by some sufficiently large and divisible power, we may assume that $e_{S^{1}}(B)=1, B$ is very ample and $K_{M} \otimes B^{\otimes(\mathrm{n}+1)} \in$ $\mathrm{Pic}^{S^{1}}(M)$ is ample and GIT-equivalent to $B$. Let us then define $S=:\left(K_{M} \otimes\right.$ $\left.B^{\otimes(\mathrm{n}+1)}\right)^{\otimes e}$, where $e=: e_{S^{1}}\left(K_{M}\right)$. By arguing as in the proof of Lemma 6.1] we may also suppose without loss of generality that $H^{0}(M, S)^{S^{1}} \neq\{0\}$. Let us choose $\sigma \in H^{0}(M, S)^{S^{1}} \neq\{0\}, \sigma \neq 0$.

For $p \geq 0$, let $R_{p}=: L^{\otimes p} \otimes S^{-1} \in \operatorname{Pic}^{S^{1}}(M)$. By Proposition 6.2 and Remark 6.2, there exists $p \gg 0$ prime with $e_{S^{1}}(L)$ such that $\operatorname{vol}_{\mu}\left(R_{p}\right)>$ $p^{\mathrm{n}-\mathrm{g}}\left(\operatorname{vol}_{\mu}(L)-\epsilon\right)$ for every $\mu \in F$. In particular, $R_{p}$ is $S^{1}$-big; by Corollary 6.2, it is big.

Let now $\mathcal{J}=\mathcal{J}\left(\left\|R_{p}\right\|\right)$ be the asymptotic multiplier ideal of $R_{p} \mathrm{~L}$. Since $R_{p} \in \operatorname{Pic}^{S^{1}}(M), \mathcal{J} \subseteq \mathcal{O}_{M}$ is $G$-invariant. Let $\beta: \widetilde{M} \rightarrow M$ be a resolution of the blow-up of $\mathcal{J}$; we may assume that $\mu$ is given by a finite sequence of blow-ups along $S^{1}$-invariant smooth centers, and therefore it is an equivariant birational morphism of projective $S^{1}$-manifolds. Thus $\mathcal{J} \cdot \mathcal{O}_{\widetilde{M}}=$ $\mathcal{O}_{\widetilde{M}}\left(-E_{p}\right)$, for an appropriate $S^{1}$-invariantly effective divisor $E_{p} \subseteq \widetilde{M}$. Set $A_{p}=: \beta^{*}(L)^{\otimes p}\left(-E_{p}\right) \in \operatorname{Pic}^{S^{1}}(\widetilde{M})$.

Lemma 10.1. $A_{p}$ is globally generated (hence nef), big and $S^{1}$-big.

Proof. Since, in additive notation, $p L=K_{M}+R_{p}+n B+\left\{(e-1)\left[K_{X}+\right.\right.$ $(n+1) B]+B\}$, Corollary 11.2.13 of $\left[\mathbf{L}\right.$ implies that $L^{\otimes p} \otimes \mathcal{J}$ is globally generated. This in turn implies that $A_{p}$ is globally generated. 
Lemma 10.2. For every $\ell \geq 1$, there is an equivariant injective linear map $H^{0}\left(M, R_{p}^{\otimes \ell}\right) \rightarrow H^{0}\left(M, L^{\otimes p \ell} \otimes \mathcal{J}^{\ell}\right)$.

Proof. This follows from the same arguments as in $[\mathrm{L}$, because all the sheaves involved and the section $\sigma$ are $S^{1}$-invariant.

As in $[\mathrm{L}$, one can deduce from this an equivariant injective linear map $H^{0}\left(M, R_{p}^{\otimes \ell}\right) \rightarrow H^{0}\left(\widetilde{M}, A_{p}^{\otimes \ell}\right)$. This implies the statement of Proposition 10.1 with $A=A_{p}, E=\mathcal{O}_{\widetilde{M}}\left(E_{p}\right)$.

Q.E.D.

Since when passing to a resolution one might lose control of the codimension of unstable loci, we may not resort directly to the equivariant Kodaira Lemma to complete the proof of the Theorem by analogy with the action-free case. We shall therefore adopt an ad hoc argument.

Proposition 10.2. A decomposition as in the statement of Theorem 10.1 exists, if we only require $A \in \mathrm{Pic}^{S^{1}}(\widetilde{M})$ to be ample, big and $S^{1}$-big.

Proof. Let $\widetilde{M}, A=A_{p}, E, p$ be as in Proposition 10.1 Let $\varphi_{k}: \widetilde{M} \rightarrow$ $\mathbb{P} H^{0}\left(\widetilde{M}, A_{p}^{\otimes k}\right)^{*}$ be the $S^{1}$-equivariant projective morphism induced by the linear series $\left|A_{p}^{\otimes k}\right|, k \geq 1$. Let $\widetilde{M}_{k}=: \varphi_{k}(M), H_{k}=\mathcal{O}_{M_{k}}(1)$. For $k \gg 0$, the projective morphisms $\varphi_{k}: \widetilde{M} \rightarrow \widetilde{M}_{k}$ stabilize to an $S^{1}$-equivariant algebraic fibre space $\varphi_{\infty}: \widetilde{M} \rightarrow \widetilde{M}_{\infty}$, and $\varphi_{k}^{*}\left(H_{k}\right)=A^{\otimes k}$ for every $k \geq 0$. One can deduce that there exists $H \in \operatorname{Pic}^{S^{1}}\left(\widetilde{M}_{\infty}\right)$ ample and such that $\varphi_{\infty}^{*}(H)=A$.

By composing a finite sequence of blow-ups along $S^{1}$-invariant smooth centers, we can find an $S^{1}$-equivariant resolution of singularities of the inverse birational map $\varphi_{\infty}^{-1}, \widetilde{M} \stackrel{\psi}{\leftarrow} \widehat{M}_{\infty} \stackrel{\phi}{\rightarrow} \widetilde{M}_{\infty}$. In particular, $\phi=\phi_{\infty} \circ \psi$. It follows that there exists an $S^{1}$-invariantly effective divisor $F \subseteq \widetilde{M}_{\infty}$ such that $\psi^{*}\left(A^{\otimes k}\right)(-F) \in \operatorname{Pic}^{S^{1}}\left(\widetilde{M}_{\infty}\right)$ is ample for every $k \gg 0$. Suppose that $F=\sum_{i} a_{i} F_{i}$, where each $a_{i}>0$ and $F_{i} \subseteq F$ is irreducible.

Lemma 10.3. We can find $k_{0}$ (divisible by $e_{S^{1}}(A)=e_{S^{1}}(L)$ ) such that

$$
H^{0}\left(\widehat{M}_{\infty}, \psi^{*}\left(A_{p}\right)^{\otimes k_{0}}(-F)\right)^{S^{1}} \neq 0
$$

Proof of Lemma 10.3. By taking tensor products, we are reduced to proving the statement in the case where $F$ is reduced and irreducible, i.e., one of the $F_{i}$ 's. Since $F$ is $S^{1}$-invariantly effective, we have for every $l \geq 0$ an equivariant short exact sequence of sheaves on $\widehat{M}_{\infty}$,

$$
0 \rightarrow \psi^{*}(A)^{\otimes l}(-F) \longrightarrow \psi^{*}(A)^{\otimes l} \longrightarrow \psi^{*}(A)^{\otimes l} \otimes \mathcal{O}_{F} \rightarrow 0 .
$$

Now $\operatorname{vol}_{0}\left(\psi^{*}(A)\right)=\operatorname{vol}_{0}(A)>0$, while: 
Claim 10.1. For some constant $C>0$, we have

$$
\operatorname{dim} H^{0}\left(F, \psi^{*}(A)^{\otimes k} \otimes \mathcal{O}_{F}\right)^{G} \leq C k^{\mathrm{n}-2}
$$

for $k \gg 0$.

Proof. We have $\psi^{*}(A)=\phi^{*}(H)$. Let $S=: \psi(F) \subseteq \widetilde{M}_{\infty}, \gamma: F \rightarrow S$ the induced morphism. Thus $S$ is an $S^{1}$-invariant irreducible subvariety of $\widetilde{M}_{\infty}$, and $\operatorname{dim}(S) \leq \mathrm{n}-2$. By the projection formula and a free resolution of $\gamma_{*} \mathcal{O}_{F}$ by powers of $H$, we are reduced to proving that $\operatorname{dim} H^{0}\left(S, H^{k} \otimes \mathcal{O}_{S}\right)^{S^{1}} \leq$ $C k^{\mathrm{n}-2}$, and this is obvious by dimension reasons.

The statement of Lemma [10.3 then follows by taking global sections in (33).

Q.E.D.

Now let us set $\widetilde{\beta}=: \beta \circ \psi: \widehat{M}_{\infty} \rightarrow M$. Then, for every $k \geq 0$ we have:

$$
\begin{aligned}
\widetilde{\beta}^{*}(L)^{\otimes p^{k}} & =\psi^{*}(A)^{\otimes p^{k-1}} \otimes \psi^{*}(E)^{\otimes p^{k-1}} \\
& =\psi^{*}(A)^{\otimes\left(p^{k-1}-k_{0}\right)} \otimes \psi^{*}(A)^{\otimes k_{0}}(-F) \otimes\left(F \otimes \psi^{*}(E)^{\otimes p^{k-1}}\right) \\
& =\widetilde{A}_{k} \otimes\left(F \otimes \psi^{*}(E)^{\otimes p^{k-1}}\right),
\end{aligned}
$$

where $\widetilde{A}_{k}=: \psi^{*}(A)^{\otimes\left(p^{k-1}-k_{0}\right)} \otimes\left(\psi^{*}(A)^{k_{0}}(-F)\right)$. By construction, $\widetilde{A}_{k}$ is ample.

Lemma 10.4. Fix $\varepsilon>0$. If $k \gg 0$, we have

$$
\operatorname{vol}_{\mu}\left(\widetilde{A}_{k}\right) \geq\left(p^{k}\right)^{\mathrm{n}-1}\left(\operatorname{vol}_{\mu}(L)-\varepsilon\right)
$$

Proof. By Lemma 10.3, for every $\mu \in F$ we have

$$
\begin{aligned}
\operatorname{vol}_{\mu}\left(\widetilde{A}_{k}\right) & \geq \operatorname{vol}_{\mu}\left(A^{\otimes p^{k-1}-k_{0}}\right) \\
& =\left(p^{k-1}-k_{0}\right)^{\mathrm{n}-1} \operatorname{vol}_{\mu}\left(A_{p}\right) \\
& \geq\left(p^{k-1}-k_{0}\right)^{\mathrm{n}-1} p^{\mathrm{n}-1}\left(\operatorname{vol}_{\mu}(L)-\epsilon\right) \\
& =\left(p^{k}\right)^{\mathrm{n}-1}\left(1-\frac{k_{0}}{p^{k-1}}\right)^{\mathrm{n}-1}\left(\operatorname{vol}_{\mu}(L)-\epsilon\right) \\
& \geq\left(p^{k}\right)^{\mathrm{n}-1}\left(\operatorname{vol}_{\mu}(L)-\epsilon^{\prime}\right)
\end{aligned}
$$

where $\epsilon^{\prime}$ can be made arbitrarily small by taking $0<\epsilon \ll 1$ and $k \gg 0$. 
This completes the proof of Proposition 10.2

Q.E.D.

From now on, we shall simplify our notation and set $M^{\prime}=\widehat{M}_{\infty}, \beta^{\prime}=\widetilde{\beta}$ : $M^{\prime} \rightarrow M, A^{\prime}=\widetilde{A}_{k}$, and simply replace $p^{k}$ with $p$. Thus, we have $\left(\beta^{\prime}\right)^{*}(L)^{\otimes p}=$ $A^{\prime} \otimes R$, for some $G$-invariantly effective $R$, and $\operatorname{vol}_{\mu}\left(A^{\prime}\right) \geq p^{\mathrm{n}-1}\left(\operatorname{vol}_{\mu}(L)-\epsilon\right)$ for every $\mu \in F$.

Proposition 10.3. The exists $B^{\prime} \in \operatorname{Pic}^{S^{1}}\left(M^{\prime}\right)$ ample and with non-empty stable locus: $\left(M^{\prime}\right)^{\mathrm{s}}\left(B^{\prime}\right) \neq \emptyset$.

Proof of Proposition 10.3. Let $H \in \mathrm{Pic}^{S^{1}}\left(M^{\prime}\right)$ be any ample Hermitian $S^{1}$-linearized line bundle, with associated moment map $\Phi_{H}: M^{\prime} \rightarrow \mathbb{R}$ (by (6), the moment maps are uniquely associated to the invariant Hermitian structure and the linearization). Let $\Phi_{B}: M \rightarrow \mathbb{R}$ be a moment map for $B$, with respect to an appropriate invariant Hermitian structure. By the regularity of $B, \Phi_{B}^{-1}(0) \neq \emptyset$, and 0 is a regular value of $\Phi_{B}$. Let us define $B_{k}=:\left(\beta^{\prime}\right)^{*}(B) \otimes H \in \operatorname{Pic}^{S^{1}}\left(M^{\prime}\right)$. Then $B_{k}$ has a product invariant Hermitian structure, with associated moment map

$$
\Phi_{B_{k}}=k \cdot\left(\Phi_{B} \circ \beta^{\prime}\right)+\Phi_{H}=k\left[\left(\Phi_{B} \circ \beta^{\prime}\right)+\frac{1}{k} \Phi_{H}\right] .
$$

Since $\beta^{\prime}$ is a proper birational morphism, the image of its exceptional locus is an invariant projective subvariety $Z \subseteq M$. Thus $\beta^{\prime}$ is an isomorpism over a dense open subset of $\Phi_{B}^{-1}(0)$, so that we can find $m \in \Phi_{B}^{-1}(0)$ and an analytic neighbourhood $M \supseteq U \ni m$ whose closure $\bar{U}$ has positive distance from $Z$. Let us identify $\bar{U}$ with its inverse image in $M^{\prime}$. In view of (34), for $k$ sufficiently large $\Phi_{B_{k}}$ is submersive at some point $m^{\prime} \in \Phi_{B_{k}}^{-1}(0) \cap \bar{U} \neq \emptyset$ near $m$. The proof of Proposition 10.3 is then completed by the following:

Lemma 10.5. Let $N$ be a projective $G$-manifold, and suppose $E \in \operatorname{Pic}^{G}(N)$ is ample. Let $h=h_{E}$ be a $G$-invariant Hermitian metric on $E$, and let $\Phi_{E}=\Phi_{A, h}$ be the induced moment map (in the sense of (6)). Suppose that:

- the normalized curvature form $\Omega$ of the associated compatible connection is Kähler;

- $\Phi_{E}^{-1}(0) \neq \emptyset$, and $\Phi_{E}$ is submersive at some $m \in \Phi_{E}^{-1}(0)$.

Then $N^{\mathrm{s}}(E) \neq \emptyset$. 
Proof of Lemma 10.5, $h$ and $\Omega$ induce unitary structures on $H^{0}\left(N, E^{\otimes k}\right)$, $k \geq 0$. Let $\Omega_{k}$ be the associated Fubini-Study form on $\mathbb{P} H^{0}\left(N, E^{\otimes k}\right)^{*}$, and let $\varphi_{k}: M \rightarrow \mathbb{P} H^{0}\left(N, E^{\otimes k}\right)^{*}$ be the $G$-equivariant projective embedding induced by the linear series $\left|E^{\otimes k}\right|, k \gg 0$. Let $\Phi_{k}: \mathbb{P} H^{0}\left(N, E^{\otimes k}\right)^{*} \rightarrow \mathfrak{g}^{*}$ be the moment map for the $G$-action.

By a well-known theorem of Tian [Ti] and Zelditch [Z], $\varphi_{k}$ is asymptotically isometric, in the sense that $\Omega-\frac{1}{k} \varphi_{k}^{*}\left(\Omega_{k}\right)=O\left(\frac{1}{k}\right)$ in $\mathcal{C}^{l}$-norm, for every l. Therefore, if $k \gg 0$ we also have $\Phi-\frac{1}{k} \Phi_{k} \circ \varphi_{k}=O\left(\frac{1}{k}\right)$ in $\mathcal{C}^{l}$-norm, for every $l$.

It then follows that, for $k \gg 0,\left(\Phi_{k} \circ \varphi_{k}\right)^{-1}(0) \neq \emptyset$, and that $\Phi_{k} \circ \varphi_{k}$ is submersive at some $m^{\prime} \in\left(\Phi_{k} \circ \varphi_{k}\right)^{-1}(0)$ close to $m$. Thus it suffices to prove statement is true when $\Phi$ is a projectively induced moment map. This in turn reduces the problem to proving it for a linear action on $\mathbb{P}^{N}$, where the statement is a direct consequence of the numerical criterion.

This completes the proof of Proposition 10.3.

Q.E.D.

To complete the proof of Theorem 10.1, we now need only compose $\beta^{\prime}$ with a Kirwan approximation, so as to assume that there exist an ample regular line bundle on $M^{\prime}$. That the factorization can be lifted with the stated properties follows by the same arguments as in the proof of Proposition 10.2.

Corollary 10.1. Let $M$ be a projective $S^{1}$-manifold, and suppose that there exists a regular and ample $B \in \operatorname{Pic}^{S^{1}}(M)$ such that $\operatorname{codim}\left(M^{\mathrm{u}}(B)\right) \geq 2$. Let $\xi \in \mathrm{NS}^{S^{1}}(M)$ be an $S^{1}$-big class and fix $\epsilon>0$. Then there exists an equivariant modification $\beta: \tilde{M} \rightarrow M$ (depending on $\xi, F$ and $\epsilon$ ) and classes $a \epsilon$ $\overline{C^{G}(\widetilde{M})}, e \in \operatorname{Eff}^{S^{1}}(\widetilde{M})$ such that $\beta^{*}(\xi)=a+e$ and $\operatorname{vol}_{0}(a) \geq \operatorname{vol}_{0}\left(\mu^{*}(\xi)\right)-\epsilon$; a may be assumed to represent an ample line bundle.

\section{References}

[BS] L. Boutet de Monvel, J. Sjöstrand, Sur la singularité des noyaux de Bergman et de Szegö, Astérisque 34-35 (1976), 123-164

[B] M. Brion, Sur les modules de covariants, Ann. Sci. École Norm. Sup. 26 (1993), 1-21

[BD] M. Brion, J. Dixmier, Comportément asymptotique des dimensions des covariants, Bull. Soc. Math. Fr. 119 (1991), 217-230

[DEL] J.-P. Demailly, L. Ein, R. Lazarsfeld, A subadditivity property of multiplier ideals, Michigan Math. J. 48 (2000), 137-156 
[DH] I. V. Dolgachev, Y. Hu, Variation of geometric invariant theory quotients. With an appendix by N. Ressayre. Inst. Hautes Études Sci. Publ. Math. 87 (1998), 5-56

[EH] S. Encinas, H. Hauser, Strong resolution of singularities in characteristic zero, Comment. Math. Helv. 77 (2002), no. 4, 821-845

[EV] S. Encinas, O. Villamayor, A new proof of desingularization over fields of characteristic zero, Proceedings of the International Conference on Algebraic Geometry and Singularities (Sevilla, 2001), Rev. Mat. Iberoamericana 19 (2003), no. 2, 339-353

[F] T. Fujita, Approximating Zariski decomposition of big line bundles, Kodai. Math. J. 17 (1994), no. 1, 1-3

[G] M. J. Gotay, On coisotropic embeddings of presymplectic manifolds, Proc. Am. Math. Soc. 84 (1982), 111-114

[GH] P. Griffiths, J. Harris, Principles of algebraic geometry, Wiley Classics Library, John Wiley \& Sons, Inc., New York, 1994

[GGK] V. Guillemin, V. Ginzburg, Y. Karshon Moment maps, cobordism, and Hamiltonian group actions, Mathematical Surveys and Monographs 98, A.M.S. (2002)

[GS1] V. Guillemin, S. Sternberg, Geometric quantization and multiplicities of group representations, Inv. Math. 67 (1982), 515-538

[GS3] V. Guillemin, S. Sternberg, Symplectic techniques in physics, Cambridge University Press 1984

[Ka] T. Kawasaki, The Riemann-Roch theorem for complex V-manifolds, Osaka J. Mat. 16 (1979), 151-157

[Ki1] F. Kirwan, Cohomology of quotients in symplectic and algebraic geometry, Mathematical Notes 31, Princeton University Press, Princeton, NJ, 1984

[Ki2] F. Kirwan, Partial desingularizations of quotients of nonsingular varieties and their Betti numbers, Ann. of Math. 122 (1985), 41-85

[Ki3] F. Kirwan, Momentum maps and reduction in algebraic geometry, Differential Geom. Appl. 9 (1998), no. 1-2, 135-171 
[JK] L. Jeffrey, F. Kirwan, Localization and the quantization conjecture, Topology 36 (1997), no. 3, 647-693

[L] R. Lazarsfeld, Positivity in Algebraic Geometry I and II, Springer 2004

[M1] E. Meinrenken, On Riemann-Roch formulas for multiplicities, Journal of the A.M.S. 9 (1996), 373-389.

[M2] E. Meinrenken, Symplectic surgery and the Spin ${ }^{c}$-Dirac operator, Adv. Math. 134 (1998), no. 2, 240-277.

[MS] E. Meinrenken, R. Sjamaar Singular reduction and quantization, Topology 38 (1999), no. 4, 699-762

[P1] R. Paoletti, The asymptotic growth of equivariant sections of positive and big line bundles, to appear in Rocky Mountain J. Math.

[P2] R. Paoletti, Moment maps and equivariant Szegö kernels, J. Symplectic Geom. 2 (2003), no. 1, 133 - 175

[S] R. Sjamaar, Symplectic reduction and Riemann-Roch formulas for multiplicities, Bull. Amer. Math. Soc. (N.S.) 33 (1996), no. 3, 327338

[Th] M. Thaddeus, Geometric invariant theory and flips, J. Amer. Math. Soc. 9 (1996), no. 3, 691-723

[Ti] G. Tian, On a set of polarized Kähler metrics on algebraic manifolds, J. Differential Geom. 32 (1990), no. 1, 99-130

[Z] S. Zelditch, Szegö kernels and a theorem of Tian, Int. Math. Res. Not. 6 (1998), 317-331 